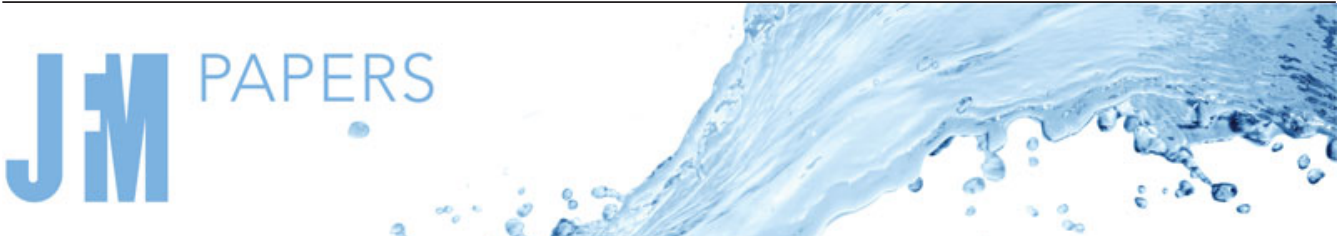

\section{Spatially evolving cascades in temporal planar jets}

\author{
A. Cimarelli ${ }^{1}$, J.-P. Mollicone ${ }^{2}$, M. van Reeuwijk ${ }^{2}$ and E. De Angelis ${ }^{3,4}, \dagger$ \\ ${ }^{1}$ DIEF, University of Modena and Reggio Emilia, 41125 Modena, Italy \\ ${ }^{2}$ Department of Civil and Environmental Engineering, Imperial College, SW7 2AZ London, UK \\ ${ }^{3}$ School of Engineering, Cardiff University, Cardiff CF24 3AA, UK \\ ${ }^{4}$ DIN, Università di Bologna, Forlì 47121, Italy
}

(Received 19 June 2020; revised 2 October 2020; accepted 4 November 2020)

Starting from an alternative decomposition of the turbulent field, a multi-dimensional statistical formalism for the description and understanding of turbulence in free-shear flows is proposed and applied to the symmetries of planar temporal jets. The theoretical framework is based on the exact equation for the second-order moment of the two-point velocity increment and allows us to trace, for the first time, the spatially evolving cascade processes at the basis of turbulence mixing and entrainment. Fascinating reverse energy cascade mechanisms are found to be responsible for the generation of long and wide structures in the interface region. Analogously to two-dimensional turbulence, the energy provided by these spatially ascending reverse cascades is found to be eventually dissipated by viscosity at large scales through friction shearing processes involving a thin cross-flow layer of these large-scale structures. Finally, the external non-turbulent region of the jet is also found to be active from an energetic point of view. It is found that pressure-mediated non-local phenomena of displacement of almost quiescent fluid give rise to non-turbulent fluctuations that in time, through transitional mechanisms, would contribute to the growth of the turbulent jet. Overall, the unexpected paths taken by the scale-energy flux in the combined physical/scale space, which are a substantial novelty with respect to known descriptions of turbulent mixing and entrainment, may have major repercussions on our theoretical understanding and modelling, as anticipated here by reduced equations capable of giving a simple scale-dependent description of the rich dynamics of the flow.

Key words: jets, turbulence theory, turbulent mixing

$†$ Email address for correspondence: deangelise@ cardiff.ac.uk

(C) The Author(s), 2021. Published by Cambridge University Press. This is an Open Access article, distributed under the terms of the Creative Commons Attribution licence (http://creativecommons.org/ licenses/by/4.0/), which permits unrestricted re-use, distribution, and reproduction in any medium, provided the original work is properly cited. 


\section{A. Cimarelli and others}

\section{Introduction}

According to the Kolmogorov's theory, the prominent feature of high-Reynolds-number turbulent flows is the energy transfer from large to small scales believed to universally occur in the inertial subrange. Kolmogorov's groundbreaking intuition was reducing the complex problem of turbulence to its essential features, by assuming homogeneity and isotropy. In these conditions, the main process is the transfer of energy among scales which is described by a single scalar quantity, the average dissipation rate. In this view, the velocity increment between two points is a central object. Energy balance prescribes its third-order moment to be linear in the separation $r$ and proportional to dissipation.

In fact, actual turbulent flows have a much richer physics, involving, beyond energy transfer in the space of scales, inhomogeneous and anisotropic processes such as spatial fluxes and turbulence production. These phenomena simply arise from the fact that every turbulent flow in reality has boundaries that, when not walls, are called turbulent interfaces. Starting from the work of Corrsin \& Kistler (1955), interfacial processes occurring between flow regions at different turbulence levels triggered the interest of many experimental, numerical and theoretical researches. Indeed, many types of turbulent flows in nature and engineering are characterized by surfaces such as iso-velocity, iso-vorticity and iso-concentration interfaces. The fluid transport process across the interface, commonly referred to as turbulent entrainment, directly affects the mixing of turbulence and controls the rate at which turbulent regions grow, thus governing the exchanges of mass, momentum, heat and species; see da Silva et al. (2014) and references therein.

The processes of turbulence entrainment and mixing take origin from the large-scale structures of turbulence in the core flow region (Hussain 1986). A spatially evolving cascade process is then initiated in the turbulent core whose final stage is the small-scale motions populating the convoluted turbulent interface where viscous mechanisms dominate (da Silva \& Mètais 2002). Indeed, it is well known that the main mechanism by which non-turbulent irrotational fluid becomes turbulent by crossing the interface is based on small-scale motions dominated by viscous diffusion processes. By adapting themselves to the flow background imposed by the large-scale features of the turbulent core, these small-scale phenomena represent the last stage of the spatially evolving cascade process of entrainment and mixing. The duality of these processes legitimates researches that alternatively concentrate to the large scales and to the small scales, the first and last stage of the process (Sreenivasan, Ramshankar \& Meneveau 1989). As a consequence, the related theories are spurious results of the observables used and, hence, are difficult to reconcile. To overcome this duality, the theoretical framework of the generalized Kolmogorov equation (Hill 2002; Dubrulle 2019) is here developed and applied. The formalism is based on the second-order moment of the two-point velocity increment. The structure of the equation, in the form of a divergence of a scale-energy flux, allows us to reconcile, for the first time, the large- and small-scale features of the spatially evolving cascade process of entrainment and mixing by tracing the scale-energy paths in the augmented space of scales and positions.

The proposed formalism strictly connects to turbulence theories and closures. As an example, the generalized Komogorov equation has been used in Cimarelli, De Angelis \& Casciola (2013) to derive a reduced description of the reverse energy cascade in wall turbulence. This reduced theory has been used in Cimarelli \& De Angelis $(2012,2014)$ to develop new physically based modelling approaches for large-eddy simulation. Indeed, as shown in Germano $(2007 a, b)$, the second-order moment of the two-point velocity increment is strictly related to turbulent stresses at different scales and can be directly 


\section{Spatially evolving cascades in temporal planar jets}

used to derive anisotropic and nonlinear turbulence closures as highlighted in Cimarelli, Abbà \& Germano (2019).

In closing the introduction to the work, it is worth noting that a previous attempt to address the multiscale features of turbulent mixing has been already performed in Cimarelli et al. (2015a) by analysing the spectral enstrophy budget equation in a shear-less flow with turbulent/non-turbulent interface. The joint description of scales and positions provided by the spectral enstrophy budget equation allowed us to measure and understand interesting aspects of the turbulent entrainment. However, the spectral decomposition does not allow for a definition of scales in statistically inhomogeneous directions and, hence, the approach used lacks a distinction between spatial fluxes and scale-space fluxes in these directions. On the contrary, the scale decomposition in the generalized Kolmogorov equation is performed in physical space, thus allowing us to also define scales in the inhomogeneous directions and to identify the related fluxes.

The paper is organized as follows. The details of the simulations performed are described in $\S 2$. The theoretical framework is presented in $\S 3$ and applied to the numerical database in $\S \S 4,5$ and 6 where the main results of the work are described. The work is closed by concluding remarks in $\S 7$ and by an appendix A where the self-similarity of the flow is exploited in detail.

\section{Turbulent temporal jet}

Turbulent plane jets can be understood as a paradigm for free-shear flows. Indeed, the flow is dominated by the presence of an inhomogeneous mean streamwise velocity profile and by the interaction of two well-separated range of scales, i.e. large-scale vortices originating from Kelvin-Helmholtz instabilities and small-scale fluctuations due to the development of turbulence. Hence, several features of these type of flows can be understood as universal being shared with other free-shear flows such as round jets, mixing layers and wakes. A further step towards the study of the essential features of free-shear flows is to consider the evolution of plane turbulent jets in time rather than in space. This choice allows us to recover a statistical homogeneity in space while loosing that in time. This simple exchange of statistical symmetries allows for a simpler formulation of the problem since the statistical formalisms representing the inhomogeneity in time are represented by single processes, e.g. momentum and energy flux in time, and, hence, are far less complex than the formalisms representing inhomogeneity in space which involve multiple physical processes, e.g. momentum flux in space, viscous, turbulent and pressure energy fluxes in space and turbulence production due to mean velocity gradient in space. For these reasons, direct numerical simulations of temporal jets represent a very useful tool for the analysis of the essential features of free-shear flows (da Silva \& Pereira 2008; van Reeuwijk \& Holzner 2014).

In the present work, we consider direct numerical simulation data of a temporal plane jet performed by solving the Navier-Stokes equations

$$
\left.\begin{array}{c}
\frac{\partial u_{i}}{\partial x_{i}}=0 \\
=-\frac{1}{\rho} \frac{\partial p}{\partial x_{i}}+v \frac{\partial^{2} u_{i}}{\partial x_{j} \partial x_{j}}
\end{array}\right\}
$$

by means of a numerical code based on a fourth-order-accurate spatial discretization and a third-order Adams-Bashforth scheme for time integration; see Craske \& van Reeuwijk (2015) and Verstappen \& Veldman (2003). In (2.1) index $i=1,2,3$ corresponds to the 


\section{A. Cimarelli and others}

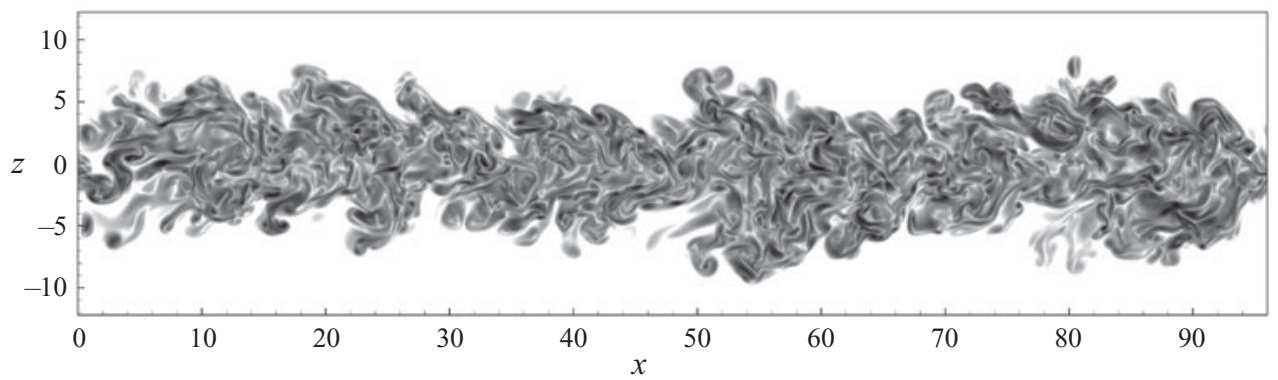

Figure 1. Direct numerical simulation of a planar temporal jet. Instantaneous flow realization at $t=120$ shown by means of iso-contours of the enstrophy field. Contour colours follow an exponential distribution. The flow domain shown is truncated in $z$ for readability reasons.

streamwise, spanwise and cross-flow $(u, v, w)$ velocities and $(x, y, z)$ directions, $\rho$ is the density and $v$ is the kinematic viscosity. The initial condition is a fluid layer that is quiescent except for a thin region $-H<z<H$ where the streamwise velocity is non-zero and homogeneously distributed in the streamwise and spanwise directions,

$$
u(x, y, z, 0)=\frac{U_{0}}{2}\left[1+\tanh \left(\frac{H-|z|}{2 \sigma_{0}}\right)\right],
$$

where $\sigma_{0}=2 H / 35$ is the initial momentum thickness. In order to facilitate a rapid transition to turbulence, a perturbation consisting of a uniform random noise is superimposed, its intensity is $4 \%$ the maximum initial velocity.

The flow problem considered here is for a Reynolds number $R e=U_{0} H / v=1000$; see figure 1 for a view of the enstrophy field. The computational domain is a cuboid of size $96 H \times 96 H \times 48 H$ and has been discretized by using $2304 \times 2304 \times 960$ elements. A variable time step has been used for the temporal integration in order to obtain a condition $C F L<0.3$. Three simulations have been performed. Every simulation is started from the same initial conditions (2.2) except for the perturbation superimposed. Hence, these simulations allow us to have statistically independent flow realizations and have been used to improve the statistical convergence of the data by using the ensemble average. From the initial conditions, the flow field is let to freely evolve in time. The total integration time for each simulation is $t=160$. When not specifically stated, in what follows variables are presented dimensionless by using $H$ for lengths and $H / U_{0}$ for times.

After an initial transition when the system develops turbulence, the flow decays and approaches a self-similar regime (Redford, Castro \& Coleman 2012; Djenidi et al. 2016) characterized by a constant Taylor Reynolds number, $R e_{\lambda}=\sqrt{2 k_{c l} / 3} \lambda_{c l} / v=50$, where $k_{c l}$ is the centreline turbulent kinetic energy and $\lambda=\sqrt{10 v k_{c l} / \epsilon_{c l}}$ is the Taylor microscale with $\epsilon_{c l}$ the centreline turbulent dissipation. By following van Reeuwijk \& Holzner (2014), the self-similar regime can be further characterized by a temporal scaling of the characteristic velocities which is proportional to $t^{-1 / 2}$ and of the characteristic lengths which is proportional to $t^{1 / 2}$. Figure 2 confirms these behaviours. In particular, it appears that turbulence has reached a dynamic equilibrium for $t>60$ when all the observables start to follow a self-similar behaviour. Figure 2 also shows the quality of the grid resolution adopted to perform the simulations. Indeed, the minimum value of the centreline Kolmogorov scale $\eta=\left(v^{3} / \epsilon_{c l}\right)^{1 / 4}$ is shown to be reached for $t=40$, leading to a grid resolution $\Delta x_{1,2} / \eta \approx 1.66$ and $\Delta x_{3} / \eta \approx 2$. The resolution employed is even more appropriate if we consider that the Kolmogorov scale increases from this minimum with 
(a)

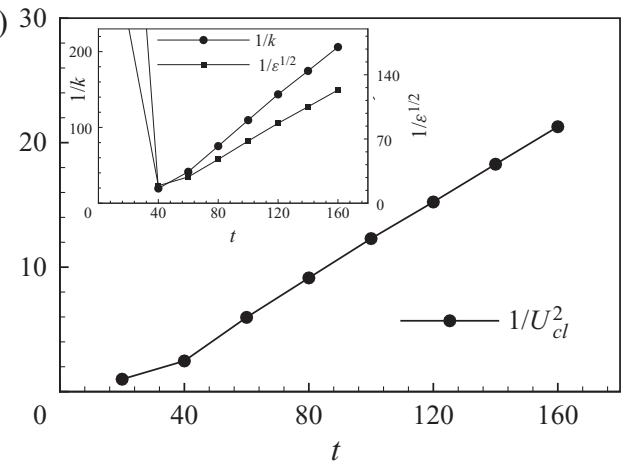

(b)

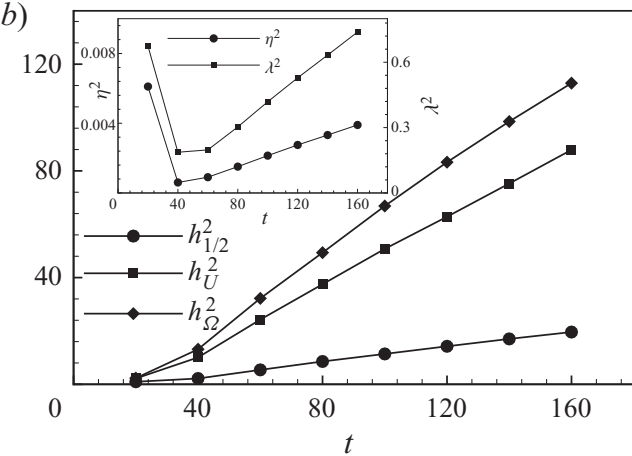

Figure 2. Temporal behaviours of relevant statistical observables. (a) Centreline mean velocity $U_{c l}(t)$. The inset shows the centreline turbulent kinetic energy $k_{c l}(t)$ and turbulent dissipation $\epsilon_{c l}(t)$. (b) Jet half-widths computed as the $50 \%$ of the mean centreline velocity, $h_{1 / 2}(t)$, and as the $2 \%$ of the mean centreline velocity and enstrophy values, $h_{U}(t)$ and $h_{\Omega}(t)$, respectively. The inset show the Kolomogorov $\eta$ and Taylor $\lambda$ scales evaluated at the centreline.

time as $\eta \sim \sqrt{t}$ and with the cross-flow position $z$ since dissipation is maximum at the centreline.

The computational domain is four times and twelve times larger in the two homogeneous directions with respect to the domain used in van Reeuwijk \& Holzner (2014) and da Silva \& Pereira (2008), respectively. This very large domain has been used in order to accurately resolve the largest scales of the flow, thereby allowing us to improve the statistical convergence because of the larger area spanned by the two homogeneous directions. Indeed, the extent of the flow domain used in the present work is a result of the study of the velocity two-point correlations from different simulations with different domain sizes. In particular, two additional simulations with the same numerical setting but different domain lengths, $24 H \times 24 H \times 36 H$ and $72 H \times 48 H \times 36 H$, have also been considered. As shown in figure 3 , the correlation coefficient of streamwise velocity,

$$
C_{u u}\left(r_{i}, z, t\right)=\frac{\left\langle u^{\prime}\left(x_{i}+r_{i}, z, t\right) u^{\prime}\left(x_{i}, z, t\right)\right\rangle}{\left\langle u^{\prime} u^{\prime}\right\rangle(z, t)},
$$

highlights significant differences in terms of correlation and anti-correlation lengths and shapes by varying the domain size. In particular, only the largest domain size appears to show a clear decorrelation of the velocity field. These results clearly highlight the need of using very large domains in order to capture the statistically relevant largest scales of the flow. It is worth pointing out that in such a flow configuration, the largest scales are represented by the large-scale vortices originating from the Kelvin-Helmholtz instability. Given the inviscid nature of this instability, we argue that such a constraint on the domain size is not mitigated by an increase of the Reynolds number.

In the present work, average quantities denoted as $\langle\cdot\rangle$ are computed by making use of the ensemble average between the different flow realizations and by taking advantage of the statistical symmetries of the flow. In particular, a spatial average is also performed in the two statistically homogeneous streamwise and spanwise directions. Furthermore, the flow exhibits a statistical symmetry in the cross-flow direction so that a transformation $z \rightarrow-z$ leaves quantities such as $\langle u\rangle$ and $\left\langle u_{i} u_{i}\right\rangle$ statistically invariant while reversing the sign of quantities such as $\langle u w\rangle$ and $\partial\langle\cdot\rangle / \partial z$. In conclusion, the average of a generic quantity 


\section{A. Cimarelli and others}
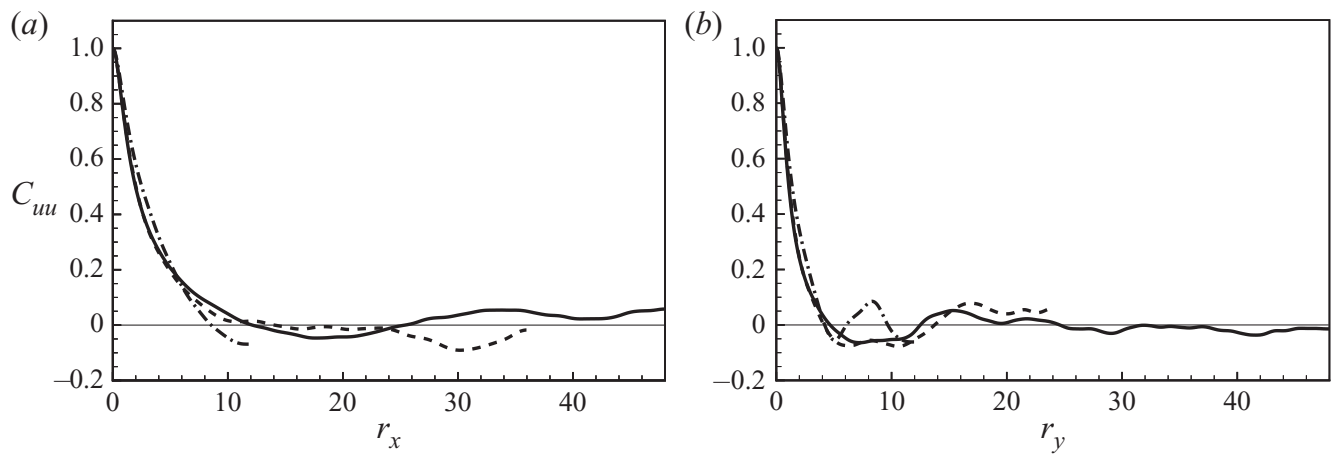

Figure 3. Two-point correlation coefficient of streamwise velocity, $C_{u u}$, evaluated at the centreline for $t=120$ as a function of the streamwise increment $r_{x}(a)$ and of the spanwise one $r_{y}(b)$. Results from three simulations with a different domain size: $24 \mathrm{H} \times 24 \mathrm{H} \times 36 \mathrm{H}$ (dashed-dotted line), $72 \mathrm{H} \times 48 \mathrm{H} \times 36 \mathrm{H}$ (dashed line) and $96 H \times 96 H \times 48 H$ (solid line).

$\beta$ is defined as

$$
\langle\beta\rangle(z, t)=\frac{1}{N} \sum_{i=1}^{N} \frac{1}{2}\left(\frac{1}{L_{x} L_{y}} \iint \beta(x, y, z, t) \mathrm{d} x \mathrm{~d} y \pm \frac{1}{L_{x} L_{y}} \iint \beta(x, y,-z, t) \mathrm{d} x \mathrm{~d} y\right),
$$

where $N=3$ is the number of flow realizations simulated and $L_{x}$ and $L_{y}$ are the dimensions of the flow domain in the streamwise and spanwise directions. In the following, the Kolmogorov length and velocity scales, $\eta$ and $u_{\eta}$, will be used for the non-dimensionalization of flow variables and will be denoted with a superscript $*$; see appendix A for the related scaling in the self-similar regime. Finally, the customary Reynolds decomposition of the flow in a mean and fluctuating field will be adopted, i.e. $u_{i}=U_{i}+u_{i}^{\prime}$.

\section{Theoretical framework}

Temporal jets are freely evolving turbulent flows characterized by a flux of momentum and kinetic energy both in space and time. In the case of planar jets, these transports in physical space occur on average only in the cross-flow direction. In these flow settings, the mean momentum equations read as

$$
\begin{gathered}
\frac{\partial U}{\partial t}+\frac{\partial}{\partial z} \underbrace{\left(\left\langle u^{\prime} w^{\prime}\right\rangle-v \frac{\partial U}{\partial z}\right)}_{\varphi_{13}=\varphi_{13}^{\text {turb }}+\varphi_{13}^{\text {visc }}}=0, \\
\frac{\partial}{\partial z} \underbrace{\left(\frac{P}{\rho}+\left\langle w^{\prime} w^{\prime}\right\rangle\right)}_{\varphi_{33}=\varphi_{33}^{\text {press }}+\varphi_{33}^{\text {turb }}}=0 .
\end{gathered}
$$

The only non-zero mean momentum is in the streamwise direction, $U$, and its flux in time is given by the unbalance of its turbulent and viscous fluxes in the cross-flow direction, $\varphi_{13}=\varphi_{13}^{\text {turb }}+\varphi_{13}^{\text {visc }} \neq 0$. On the contrary, the mean momentum in the cross-flow direction is null and turbulent and pressure fluxes in the cross-flow direction balance themselves, 


\section{Spatially evolving cascades in temporal planar jets}

$\varphi_{33}=\varphi_{33}^{\text {press }}+\varphi_{33}^{\text {turb }}=0$. As already stated, also the mean and turbulent kinetic energy is characterized by a flux in time and in the cross-flow direction. The mean kinetic energy budget reads as

$$
\frac{\partial K}{\partial t}=-\frac{\partial}{\partial z} \underbrace{\left(\left\langle u^{\prime} w^{\prime}\right\rangle U-v \frac{\partial K}{\partial z}\right)}_{\Psi_{z}=\Psi_{z}^{\text {turb }}+\Psi_{z}^{\text {visc }}} \underbrace{+\left\langle u^{\prime} w^{\prime}\right\rangle \frac{\partial U}{\partial z}}_{-P_{t}}-\underbrace{v \frac{\partial U}{\partial z} \frac{\partial U}{\partial z}}_{E},
$$

where $K=U U / 2$, while the turbulent kinetic energy budget is

$$
\frac{\partial\langle k\rangle}{\partial t}=-\frac{\partial}{\partial z} \underbrace{\left(\left\langle k w^{\prime}\right\rangle+\frac{1}{\rho}\left\langle p^{\prime} w^{\prime}\right\rangle-v \frac{\partial\langle k\rangle}{\partial z}\right)}_{\psi_{z}=\psi_{z}^{\text {turb }}+\psi_{z}^{\text {press }}+\psi_{z}^{\text {visc }}} \underbrace{-\left\langle u^{\prime} w^{\prime}\right\rangle \frac{\partial U}{\partial z}}_{P_{t}}-\underbrace{v\left\langle\frac{\partial u_{i}^{\prime}}{\partial x_{j}} \frac{\partial u_{i}^{\prime}}{\partial x_{j}}\right\rangle}_{\epsilon},
$$

where $k=u_{i}^{\prime} u_{i}^{\prime} / 2$. Contrary to the mean momentum balance equations, source and sink terms also appear together with spatial fluxes in the cross-flow direction in the kinetic energy budget equations. In particular, in the mean kinetic energy budget (3.3), only a sink term appears that is the sum of production of turbulent fluctuations, $P_{t}$, and viscous dissipation by mean shear, $E$. The unbalance of this sink with the transport due to turbulent and viscous fluxes, $\partial \Psi_{z} / \partial z$, governs the time variation of mean kinetic energy. In the turbulent kinetic energy budget (3.4), the turbulence production term $P_{t}$ is, on the contrary, a source. Hence, the sum $P_{t}-\epsilon$ can be both positive and negative, thus establishing source and sink flow regions. The energy transports due to turbulent, pressure and viscous fluxes, $\partial \psi_{z} / \partial z$, partially conform with these regions by extracting energy in the source and releasing it in the sink regions. The unbalance between source/sink and spatial transports governs the flux of turbulent kinetic energy in time.

The class of processes described by the mean momentum and energy budget equations are phenomena that occur in physical space. Hence, in the context of free-shear flows, these equations enable the study of the phenomena and positions where momentum and turbulence are developed and the transport mechanisms sustaining the spreading and entrainment of the jet. However, turbulence is known to also be characterized by phenomena taking place in the space of scales such as the turbulent cascade and, in free-shear flows, the large-scale engulfment and small-scale nibbling mechanisms of entrainment. This duality of description of the same physical phenomenon is a result of the statistical observables used for its study. The drawback is the development of different theories as spurious results of approaches that do not support the occurrence of phenomena simultaneously involving different scales and positions.

To overcome this scale/position duality, in the present work we propose the use of an alternative formalism, the so-called generalized Kolmogorov equation (Hill 2002). It is a differential equation that, in its original form valid for homogeneous and isotropic turbulence, can be traced back to Kolmogorov himself (Kolmogorov 1991a,b). The theoretical framework is based on the exact equation for the second-order moment of the velocity increment, $\delta u_{i}=u_{i}^{\prime}\left(x^{\prime \prime}, t\right)-u_{i}^{\prime}\left(x^{\prime}, t\right)$, the so-called second-order structure function, $\delta q^{2}=\delta u_{i} \delta u_{i}$ (Dubrulle 2019). The second-order structure function depends both on the separation vector $r_{i}=x_{i}^{\prime \prime}-x_{i}^{\prime}$ and on the position of the mid-point $x_{c i}=$ $\left(x_{i}^{\prime \prime}+x_{i}^{\prime}\right) / 2$. Hence, in the general case $\left\langle\delta q^{2}\right\rangle$ depends upon seven independent variables, the three coordinates of the mid-point position $\boldsymbol{x}_{\boldsymbol{c}}$, the three-dimensional scale separation $\boldsymbol{r}$ and time $t$. The generalized Kolmogorov equation has been used in several works to study the energetics of turbulence in the complete space of scales and positions, we mention only 


\section{A. Cimarelli and others}

few of them, e.g. Danaila et al. (2001), Marati, Casciola \& Piva (2004), Cimarelli et al. (2013, 2015b, 2016), Saikrishnan et al. (2012) for turbulent channel flows, Mollicone et al. (2018) for separated flows, Rincon (2006), Togni, Cimarelli \& De Angelis $(2015,2019)$ for thermally driven turbulence, Gomes-Fernandes, Ganapathisubramani \& Vassilicos (2015); Portela, Papadakis \& Vassilicos (2017) for turbulent wakes and Burattini, Antonia \& Danaila (2005), Sadeghi, Lavoie \& Pollard (2016) for round jets. Here, we extend the use of the generalized Kolmogorov equation to the symmetries of a turbulent planar temporal jet. The equation reads as

$$
\begin{aligned}
& \frac{\partial\left\langle\delta q^{2}\right\rangle}{\partial t}+\underbrace{\frac{\partial\left\langle\delta q^{2} \delta u_{i}^{\prime}\right\rangle}{\partial r_{i}}+\frac{\partial\left\langle\delta q^{2} \delta U\right\rangle}{\partial r_{x}}}_{-T_{r}}-\underbrace{2 v \frac{\partial^{2}\left\langle\delta q^{2}\right\rangle}{\partial r_{i} \partial r_{i}}}_{-D_{r}}+\underbrace{\frac{\partial\left\langle\delta q^{2} \tilde{w}^{\prime}\right\rangle}{\partial z_{c}}+\frac{2}{\rho} \frac{\partial\left\langle\delta p^{\prime} \delta w^{\prime}\right\rangle}{\partial z_{c}}-\frac{\nu}{2} \frac{\partial^{2}\left\langle\delta q^{2}\right\rangle}{\partial z_{c} \partial z_{c}}}_{-T_{z}} \\
& =\underbrace{-2\left\langle\delta u^{\prime} \delta w^{\prime}\right\rangle \widehat{\left(\frac{\partial U}{\partial z}\right)}-2\left\langle\delta u^{\prime} \tilde{w}^{\prime}\right\rangle \delta\left(\frac{\partial U}{\partial z}\right)}_{\Pi}-4 \underbrace{v\left\langle\widetilde{\left.\frac{\partial u_{i}^{\prime}}{\partial x_{j}} \frac{\partial u_{i}^{\prime}}{\partial x_{j}}\right\rangle}\right.}_{\tilde{\epsilon}},
\end{aligned}
$$

where $\sim$ denotes the two-point mean operator that, for a generic quantity, reads as $\tilde{\beta}=\left(\beta\left(x^{\prime \prime}, t\right)+\beta\left(x^{\prime}, t\right)\right) / 2$. To highlight its conservative form, (3.5) can be rewritten as

$$
\frac{\partial\left\langle\delta q^{2}\right\rangle}{\partial t}+\frac{\partial \phi_{r_{i}}}{\partial r_{i}}+\frac{\partial \phi_{z_{c}}}{\partial z_{c}}=\xi
$$

where

$$
\begin{aligned}
\frac{\partial \phi_{r_{i}}}{\partial r_{i}} & =\frac{\partial}{\partial r_{i}}\left(\phi_{r_{i}}^{\text {turb }}+\phi_{r_{i}}^{\text {mean }}+\phi_{r_{i}}^{\text {visc }}\right), \\
\frac{\partial \phi_{z_{c}}}{\partial z_{c}} & =\frac{\partial}{\partial z_{c}}\left(\phi_{z_{c}}^{\text {turb }}+\phi_{z_{c}}^{\text {press }}+\phi_{z_{c}}^{\text {visc }}\right)
\end{aligned}
$$

are the divergence of fluxes occurring in the space of scales $r_{i}$ and in physical space $z_{c}$, respectively. In particular, the scale-space flux $\phi_{r_{i}}$ is given by three contributions

$$
\phi_{r_{i}}^{\text {turb }}=\left\langle\delta q^{2} \delta u_{i}^{\prime}\right\rangle, \quad \phi_{r_{i}}^{\text {mean }}=\left\langle\delta q^{2} \delta U\right\rangle \delta_{i 1}, \quad \phi_{r_{i}}^{v i s c}=-2 \nu \frac{\partial\left\langle\delta q^{2}\right\rangle}{\partial r_{i}}
$$

due to inertial turbulent fluctuations, to mean advection and to viscous diffusion, respectively. On the other hand, the spatial flux is given by three contributions

$$
\phi_{z_{c}}^{\text {turb }}=\left\langle\delta q^{2} \tilde{w}^{\prime}\right\rangle, \quad \phi_{z_{c}}^{\text {press }}=\frac{2}{\rho}\left\langle\delta p^{\prime} \delta w^{\prime}\right\rangle, \quad \phi_{z_{c}}^{v i s c}=-\frac{v}{2} \frac{\partial\left\langle\delta q^{2}\right\rangle}{\partial z_{c}}
$$

due to inertial turbulent fluctuations, to pressure fluctuations and viscous diffusion, respectively. These transport terms are partially driven by a source term which is the balance between turbulence production and dissipation,

$$
\xi=\Pi-4 \tilde{\epsilon} .
$$

The unbalance between transports and sources governs the flux of scale energy in time, that in a symbolic form reads as

$$
\frac{\partial\left\langle\delta q^{2}\right\rangle}{\partial t}=T_{r}+D_{r}+T_{z}+\xi
$$

In line with the single-point energy budgets, the generalized Kolmogorov equation allows for the study of the source and sink regions of the flow and of the fluxes connecting 
(a)

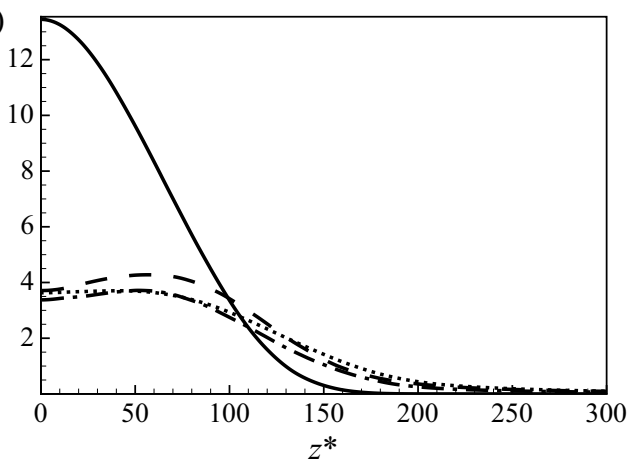

(b)

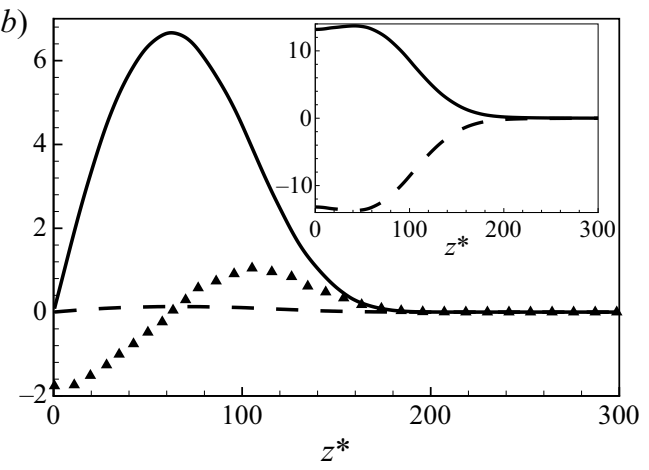

Figure 4. (a) Mean velocity and turbulent intensity profiles: $U^{*}$ (solid line), $\sqrt{\left\langle u^{\prime} u^{\prime}\right\rangle^{*}}$ (dashed line), $\sqrt{\left\langle v^{\prime} v^{\prime}\right\rangle^{*}}$ (dashed-dotted line) and $\sqrt{\left\langle w^{\prime} w^{\prime}\right\rangle^{*}}$ (dotted line). (b) Mean momentum equation. Main panel: $10 \partial U^{*} / \partial t^{*}$ (delta), mean streamwise momentum fluxes $\varphi_{13}^{\text {turb* }}$ (solid) and $\varphi_{13}^{\text {visc* }}$ (dash). Inset panel: mean cross-flow momentum fluxes $\varphi_{33}^{\text {turb* }}$ (solid) and $\varphi_{33}^{\text {press* }}$ (dash).

these regions. However, the strength of the formalism is such that all these processes are both scale and position dependent, and, as a consequence, also fluxes in the space of scales can be defined, i.e. $\phi_{r_{i}}$.

\section{Inhomogeneity and flow regions}

Before analysing the compound space of scales and positions described by the generalized Kolmogorov equation (3.5), it is useful to divide the flow in different regions characterized by well-defined physical processes. Indeed, the flow while freely evolving in time, is characterized by a strong statistical inhomogeneity in the cross-flow direction as clearly highlighted by the mean velocity and turbulent intensity profiles shown in figure $4(a)$. In particular, the flow can be idealized as characterized by a turbulent core where the mean flow and the turbulent fluctuations are intense and by an interface region where entrainment processes occur. From the mean flow point of view, both these two flow regions are characterized by a cross-flow spreading. As shown in figure 4(b), a positive turbulent flux of mean streamwise momentum in the cross-flow direction, $\varphi_{13}^{\text {turb }}>0$, is found to cover the entire flow domain. The intensity of the turbulent flux far exceeds the still positive contribution given by the viscous flux, $\varphi_{13}^{\text {visc }} \approx 0$, thus sustaining a spreading of the mean streamwise momentum profile $\varphi_{13}=\varphi_{13}^{\text {turb }}+\varphi_{13}^{\text {visc }}>0$. As a consequence, the time variation of the mean streamwise momentum is positive in the outer jet region $\partial U / \partial t>0$ and negative in the inner jet region $\partial U / \partial t<0$. The cross-over between decaying and enhancing mean momentum is $z^{*} \approx 60$, corresponding to the peak value of the turbulent flux $\varphi_{13}^{\text {turb }}$. Due to the symmetries of the flow, the mean cross-flow momentum is zero and, as shown in the inset of figure $4(b)$, a balance of positive turbulent flux, $\varphi_{33}^{\text {turb }}>0$, and negative pressure flux, $\varphi_{33}^{\text {press }}<0$, takes place.

In line with the processes governing momentum, the mean kinetic energy budget also highlights that the entire flow domain is characterized by a cross-flow spreading. As shown in the inset of figure 5, the temporal jet is characterized by a continuous flux of mean kinetic energy from its core towards the outer interface region, $\Psi_{z}=\Psi_{z}^{\text {turb }}+\Psi_{z}^{\text {visc }}>0$. Both viscous diffusion and turbulent fluxes are positive but the contribution of the turbulent flux far exceeds that due to viscosity, $\Psi_{z}^{\text {turb }} \gg \Psi_{z}^{\text {visc }}>0$. As a consequence, the turbulent transport term $\partial \Psi_{z}^{\text {turb }} / \partial z$ is the dominant term of the budget. As shown in 


\section{A. Cimarelli and others}

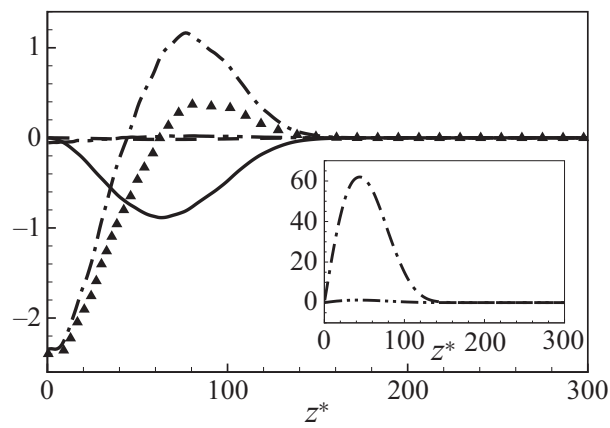

Figure 5. Mean kinetic energy budget. Main panel: $\partial K^{*} / \partial t^{*}$ (delta), $-P_{t}^{*}$ (solid), $-E^{*}$ (dash), $-\partial \Psi_{z}^{t u r b *} / \partial z^{*}$

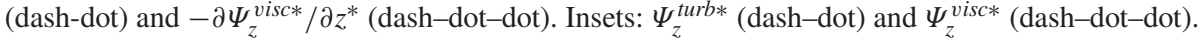

(a)

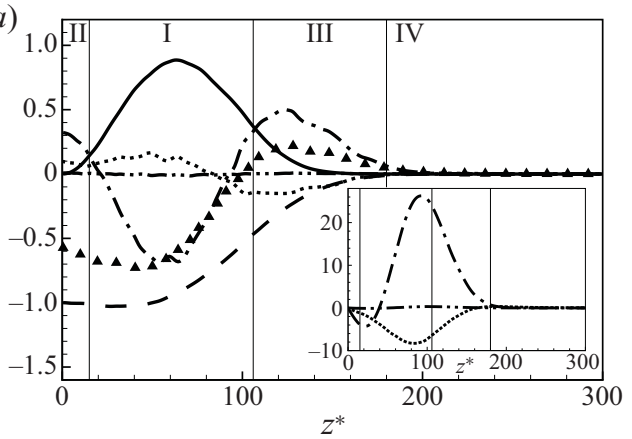

(b)

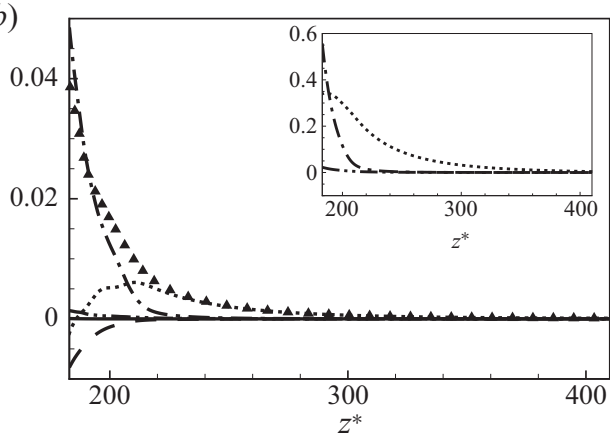

Figure 6. Turbulent kinetic energy budget. Main panels: $\partial\langle k\rangle^{*} / \partial t^{*}$ (delta), $P_{t}^{*}$ (solid), $-\epsilon^{*}$ (dash), $-\partial \psi_{z}^{\text {turb* } *} / \partial z^{*}$ (dash-dot), $-\partial \psi_{z}^{\text {press* }} / \partial z^{*}$ (dot) and $-\partial \psi_{z}^{v i s c *} / \partial z^{*}$ (dash-dot-dot). Insets: $\psi_{z}^{\text {turb* }}$ (dash-dot), $\psi_{z}^{\text {press* }}(\mathrm{dot})$ and $\psi_{z}^{\text {visc* }}$ (dash-dot-dot). The overall behaviour is shown in $(a)$ while an enlargment of the behaviour in the interface region is shown in $(b)$.

the main panel of figure 5, $\partial \Psi_{z}^{\text {turb }} / \partial z$ drains kinetic energy for $z^{*}<45$ to feed the outer region of the jet for $z^{*}>45$ with a peak intensity at $z^{*} \approx 80$. The other terms of the budget are negligible with the exception of the turbulence production term $-P_{t}$ which drains kinetic energy from the mean flow to sustain turbulent fluctuations and, hence, it is negative with a peak intensity at $z^{*} \approx 60$. These two processes of turbulence production and turbulent transport govern the behaviour of mean kinetic energy and their unbalance results in a time variation of mean kinetic energy. In accordance with the mean momentum results, the cross-over between decaying and enhancing mean kinetic energy is $z^{*} \approx 60$.

Contrary to the mean momentum and mean kinetic energy processes, the behaviour of turbulent kinetic energy allows us to characterize the flow in different flow regions depending on the physical process sustaining turbulence. As shown in figure 6(a), turbulence production $P_{t}$ is active in an intermediate region in between the core and outer part of the jet where the mean shear is maximum; see also figure 4(a). On the other hand, turbulent dissipation $-\epsilon$ shows a decrease of its intensity moving from the jet core towards the interface region. The spatial transport $-\partial \psi_{z} / \partial z$ is dominated by the turbulent and pressure terms being that the viscous diffusion is negligible almost everywhere, $\partial \psi_{z} / \partial z \approx \partial\left(\psi_{z}^{\text {turb }}+\psi_{z}^{\text {press }}\right) / \partial z$.

The overall picture is the following. In the intermediate region, $15<z^{*}<106$ (production region I), fluctuations are mainly sustained by turbulence production. 


\section{Spatially evolving cascades in temporal planar jets}

The available turbulent energy is drained by both local turbulent dissipation processes $-\epsilon$ and by turbulent transport mechanisms $-\partial \psi_{z}^{\text {turb }} / \partial z<0$ which overcomes the opposite in sign pressure transport contribution $-\partial \psi_{z}^{\text {turb }} / \partial z>0$. The balance is negative giving rise to a peak of turbulent kinetic energy decay in time within this region, $\partial\langle k\rangle / \partial t<0$. The energy subtracted by the turbulent transport is then transferred towards the inner and outer regions of the jet. In particular, for $z^{*}<15$ (inner region II) and $z^{*}>106$ (outer region III), the dominant process sustaining turbulence is the turbulent transport, $-\partial \psi_{z}^{\text {turb }} / \partial z>0$, and the budget in these two regions reduces to

$$
\frac{\partial\langle k\rangle}{\partial t} \approx-\frac{\partial}{\partial z}\left(\psi_{z}^{\text {turb }}+\psi_{z}^{\text {press }}\right)-\epsilon
$$

since production is almost negligible. The main difference between these two regions is that in the inner region II both turbulent and pressure transports are positive, $-\partial \psi_{z}^{\text {turb }} / \partial z>-\partial \psi_{z}^{\text {press }} / \partial z>0$, but the balance with dissipation is negative leading to a turbulent energy decay, $\partial\langle k\rangle / \partial t<0$. On the contrary, in the outer region III the pressure transport is negative but the turbulent transport is positive and large enough to have a positive balance with dissipation leading to $\partial\langle k\rangle / \partial t>0$ and reaching its maximum for $z^{*} \approx 125$.

Overall, we observe that, for $z^{*}<180$, the turbulent transport is more intense than the pressure one and plays a role of draining of turbulent energy from region I to feed regions II and III. Accordingly, as shown in the inset of figure 6(a), a cross-over location $z^{*} \approx 40$ can be defined splitting the flow in regions traversed by a negative turbulent flux, $\psi_{z}^{\text {turb }}<0$, directed towards the jet centreline for $z^{*}<40$ from regions traversed by a positive flux, $\psi_{z}^{\text {turb }}>0$, towards the jet interface for $z^{*}>40$. On the other hand, pressure transport drains turbulent energy in the outer region III to feed the inner regions of the jet (regions I and II). Hence, for $z^{*}<180$, the turbulent jet is entirely traversed by a negative pressure

flux, $\psi_{z}^{\text {press }}<0$, as shown in the inset of figure $6(a)$. Note that the jet interface defined as the $2 \%$ of the jet centreline enstrophy is located at $z^{*}=h_{\Omega}^{*}=171$.

Different considerations can be drawn for the external region IV, for $z^{*}>180$, i.e. further away from the putative jet interface at $z^{*}=h_{\Omega}^{*}=171$. As for the outer region III, fluctuations in this region of the flow are sustained by transport mechanisms. However, as shown in figure $6(b)$, in this case the contribution of the pressure transport turns out to be positive, $-\partial \psi_{z}^{\text {press }} / \partial z>0$ and, for $z^{*}>210$, it overcomes the contribution of the turbulent transport, i.e. $-\partial \psi_{z}^{\text {press }} / \partial z>-\partial \psi_{z}^{\text {turb }} / \partial z>0$. Interestingly, for $z^{*}>210$, the turbulent dissipation is almost null, thus suggesting that the fluctuations observed at the external region of the jet are not turbulent in nature. Hence, in this region the balance reduces to

$$
\frac{\partial\langle k\rangle}{\partial t} \approx-\frac{\partial \psi_{z}^{\text {press }}}{\partial z}>0
$$

We conjecture that such time increasing of the kinetic energy content is induced by large-scale pressure fluctuations induced by the large-scale vortex pattern of the jet reminiscent of the Kelvin-Helmholtz instability. Hence, we argue that the transport of turbulent kinetic energy also occurs in the non-turbulent region further away from the jet interface throughout non-local interactions of the large-scale jet pattern with the surrounding quiescent fluid mediated by the pressure field, $\partial \psi_{z}^{\text {press }} / \partial z$. These aspects will be further investigated in $\S 6.5$ by means of a scale-by-scale analysis. 


\section{A. Cimarelli and others}

\section{Scale-energy paths}

We now extend the analysis of the turbulent temporal planar jet to the augmented space of turbulence $\left(r_{x}, r_{y}, r_{z}, z_{c}, t\right)$ described by the generalized Kolmogorov equation (3.5). The multi-dimensionality of the approach challenges for a rational study. For this reason, we will limit the analysis to the hyper-planes $\left(r_{z}=0, t=\mathrm{const}\right)$ and $\left(r_{x}=0, t=\mathrm{const}\right)$ of the five-dimensional space of locations, scales and times. The resulting equations retain their conservative form,

$$
\begin{aligned}
& \frac{\partial \phi_{r_{x}}\left(r_{x}, r_{y}, z_{c}\right)}{\partial r_{x}}+\frac{\partial \phi_{r_{y}}\left(r_{x}, r_{y}, z_{c}\right)}{\partial r_{y}}+\frac{\partial \phi_{z_{c}}\left(r_{x}, r_{y}, z_{c}\right)}{\partial z_{c}}=\zeta_{r_{z}=0} \quad\left(r_{z}=0, t=\mathrm{const}\right), \\
& \frac{\partial \phi_{r_{y}}\left(r_{y}, r_{z}, z_{c}\right)}{\partial r_{y}}+\frac{\partial \phi_{r_{z}}\left(r_{y}, r_{z}, z_{c}\right)}{\partial r_{z}}+\frac{\partial \phi_{z_{c}}\left(r_{y}, r_{z}, z_{c}\right)}{\partial z_{c}}=\zeta_{r_{x}=0} \quad\left(r_{x}=0, t=\mathrm{const}\right),
\end{aligned}
$$

thus showing that scale-energy fluxes in the hyper-planes are driven by extended source terms,

$$
\begin{aligned}
& \zeta_{r_{z}=0}=\xi-\frac{\partial \phi_{r_{z}}}{\partial r_{z}}-\frac{\partial\left\langle\delta q^{2}\right\rangle}{\partial t}, \\
& \zeta_{r_{x}=0}=\xi-\frac{\partial \phi_{r_{x}}}{\partial r_{x}}-\frac{\partial\left\langle\delta q^{2}\right\rangle}{\partial t},
\end{aligned}
$$

which take into account the scale energy exchange with the local sink/source phenomena, $\xi$, and with the dimensions perpendicular to the hyper-plane considered, i.e.

$$
\begin{aligned}
& -\frac{\partial \phi_{r_{z}}}{\partial r_{z}}-\frac{\partial\left\langle\delta q^{2}\right\rangle}{\partial t} \quad \text { for } \quad\left(r_{z}=0, t=\mathrm{const}\right), \\
& -\frac{\partial \phi_{r_{x}}}{\partial r_{x}}-\frac{\partial\left\langle\delta q^{2}\right\rangle}{\partial t} \quad \text { for } \quad\left(r_{x}=0, t=\mathrm{const}\right) .
\end{aligned}
$$

Hence, the divergence of fluxes in the hyper-planes can be understood as the intensity of scale energy extracted/released by the flux vector field along its trajectories. In equilibrium turbulence the divergence of fluxes in the hyper-planes always balances with the sum of the local source term $\xi$ (production minus dissipation) and with the scale energy exchange to/from the scale space perpendicular to the hyper-plane itself, $\partial \phi_{r_{\perp}} / \partial r_{\perp}$, thus, $\partial\left\langle\delta q^{2}\right\rangle / \partial t=0$. In non-equilibrium turbulence such as in the temporal jet, the divergence of fluxes in the hyper-plane can exceed/subceed the sum $\xi-\partial \phi_{r_{\perp}} / \partial r_{\perp}$, thus enabling a scale-energy transfer in time $\partial\left\langle\delta q^{2}\right\rangle / \partial t \neq 0$.

\subsection{The hyper-plane $\left(r_{z}=0, t=120\right)$}

We start the analysis by considering the hyper-plane $\left(r_{z}=0, t=120\right)$. In figure 7 the trajectories of the flux vector field, $\left(\phi_{r_{x}}, \phi_{r_{y}}, \phi_{z_{c}}\right)$, coloured by the intensity of scale energy extracted/released along their route, $\partial \phi_{r_{x}} / \partial r_{x}+\partial \phi_{r_{y}} / \partial r_{y}+\partial \phi_{z_{c}} / \partial z_{c}$, are shown. The plots show that all the fluxes emerge from a well-defined point of the augmented space, $\left(r_{x}^{*}, r_{y}^{*}, z_{c}^{*}\right) \approx(90,110,60)$. As shown in figure $7(a)$ with an iso-surface of $\left\langle\delta q^{2}\right\rangle=$ $0.98\left\langle\delta q^{2}\right\rangle_{\max }$, this singularity point belongs to a range of scales and positions of the augmented space characterized by the largest scale-energy content. This energy-containing region takes a toroid shape that in physical space lies in the $z_{c}^{*} \approx 60$ plane. A well-defined band of energy-containing scales is also identified by the iso-surface of 


\section{Spatially evolving cascades in temporal planar jets}

$\left\langle\delta q^{2}\right\rangle=0.98\left\langle\delta q^{2}\right\rangle_{\max }$ that can be traced by considering the radius of revolution of this toroid shape. We find that the radius is not constant and equals $r_{y}^{*} \approx 160$ for $r_{x}^{*}=0$ and $r_{x}^{*} \approx 140$ for $r_{y}^{*}=0$, thus highlighting the anisotropy of the large energy-containing scales of the flow. As it can be grasped from the iso-contours of figures 7(b) and 7(c), this range of scales and positions is also the site of the local turbulence production mechanisms being that the source term is maximum and positive, $\xi>0$, i.e. turbulence production exceeds dissipation in this region of the augmented space. By considering that the jet half-width is $h_{\Omega}^{*}=171$ (evaluated as the location where enstrophy is $2 \%$ of the jet centreline enstrophy), we can assert that this toroidal source region of energy-containing fluctuations occurs at length scales of the same order of the integral scale of the problem.

In accordance with the above analysis, we can assert that the field of fluxes stems from the source and energy-containing region of the augmented space of scales and positions. A more detailed analysis shows that from the singularity point $\left(r_{x}^{*}, r_{y}^{*}, z_{c}^{*}\right) \approx(90,110,60)$, the fluxes bifurcate in three distinct branches approaching the $z_{c}$-distributed small-scale range, the inner region of the jet and outer regions of the jet; see figure $7(a)$. These three regions are the sink regions of the augmented space of turbulence where scale energy is eventually conveyed. Note the complex nature of the scale-energy paths towards these three regions which deserves the following separate analysis.

\subsubsection{Family of fluxes $\mathcal{A}$}

The first family of fluxes is that feeding the $z_{c}$-distributed dissipative range of small scales. This branch of fluxes while diverging from the singularity point systematically move towards smaller streamwise and spanwise scales, $\phi_{r_{x}}<0$ and $\phi_{r_{y}}<0$, respectively. As better shown in figures $7(b)$ and $7(c)$, after an initial ascension towards outer positions, $\phi_{z_{c}}>0$, this family of fluxes bends, attaining a flow towards inner positions, $\phi_{z_{c}}<0$. This picture is maintained for the entire range of inertial and energy-containing scales, $r^{*}>O(20)$, where $r^{*} \equiv\left(r_{x}^{* 2}+r_{y}^{* 2}\right)^{1 / 2}$. In fact, an interesting aspect appears in the final range of scales intercepted by the fluxes. As clearly shown in figure 7 , by entering the dissipative range of small scales, $r^{*}<O(20)$, the fluxes show a second reversal of the spatial flux towards outer positions, $\phi_{z_{c}}>0$. As a consequence, the entire $z_{c}$-distributed range of small scales is found to be intercepted by the branch of fluxes $\mathcal{A}$. The effect of spatial inhomogeneity vanishes only at very small separations where, the small-scale asymptotic is $\left(\phi_{r_{x}}, \phi_{r_{y}}, \phi_{z_{c}}\right) \sim(1,1,0) r^{*}$ (Cimarelli et al. 2013) and, hence, the fluxes become asymptotically orthogonal to the $z_{c}$-axis.

As shown by the intensity of the flux divergence reported with colours in figure 7 , the field of fluxes extracts scale energy from the large energy-containing scales of the turbulence production region I and releases it to the $z_{c}$-distributed dissipative range of small scales. The overall picture is that of a $z_{c}$-distributed dissipative range of small scales fed by an ascending direct energy cascade whose origin can be traced back to the inertial scales of the inner region and, before that, to the energy-containing scales of the production region. Hence, the behaviour of the family of fluxes $\mathcal{A}$ is entirely consistent with the picture of a Richardson turbulent energy cascade superimposed to inhomogeneous spatial redistribution processes.

We investigate here more in detail the origin of the observed inversion of the spatial flux along the paths of the family of fluxes $\mathcal{A}$. In particular, we observed that, in the inertial and energy-containing scales $r^{*}>O(20)$, the spatial flux is negative and directed towards the inner region of the jet while, in the dissipative scales $r^{*}<O(20)$, the spatial flux becomes positive thus reversing towards the outer regions of the jet. The reason of the small-scale ascending phenomenon is given by the concurrent role played by the turbulent and pressure 


\section{A. Cimarelli and others}

(a)

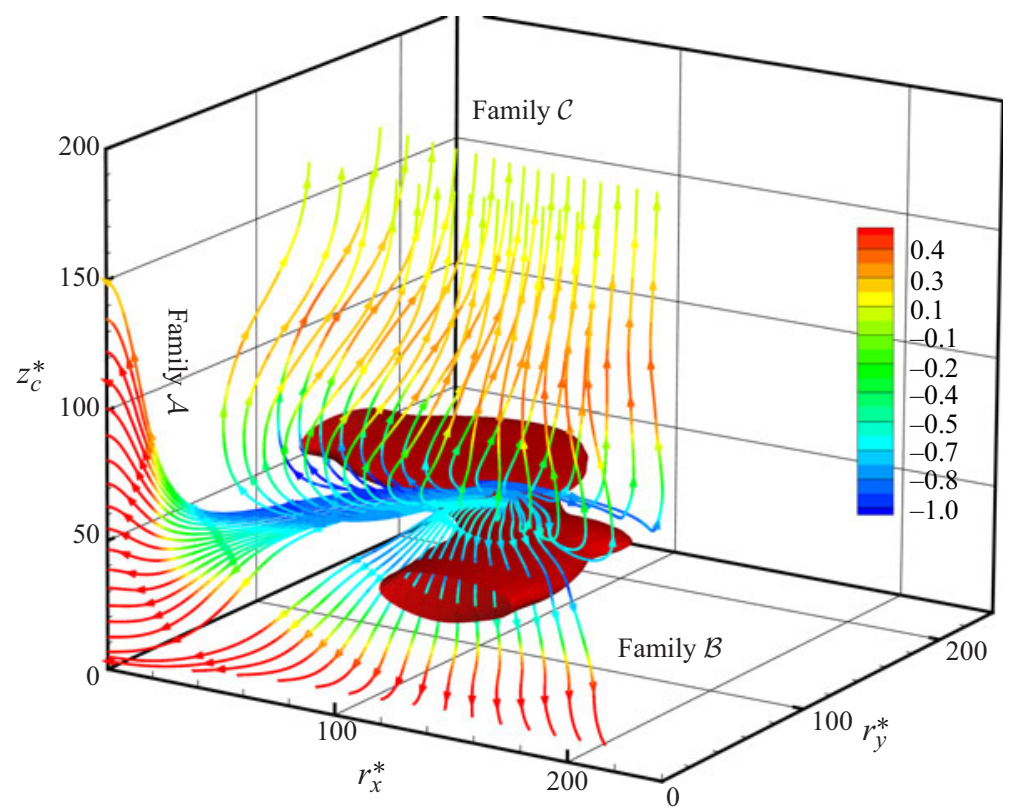

(b)

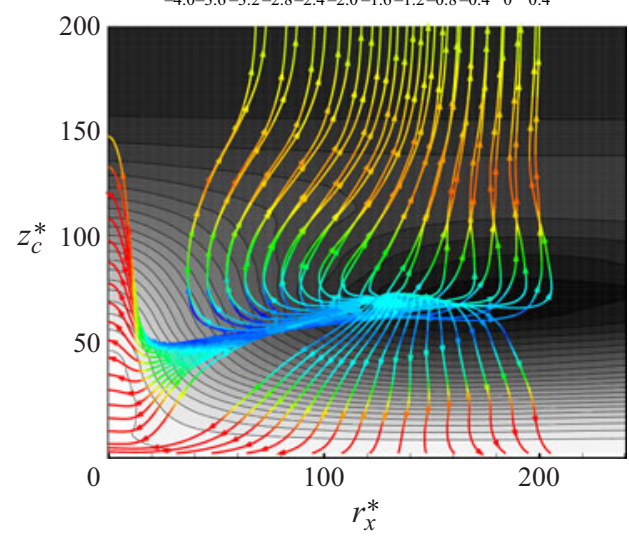

(c)

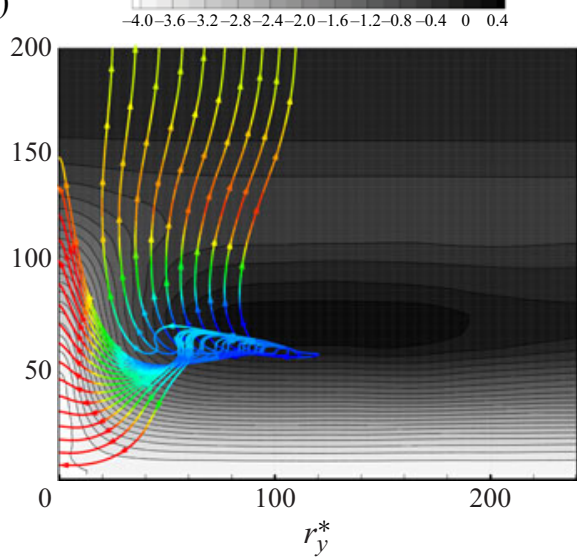

Figure 7. Paths of scale energy in the hyper-plane $r_{z}=0$. Trajectories of the flux vector field, $\left(\phi_{r_{x}}, \phi_{r_{x}}, \phi_{z_{c}}\right)$, coloured by the intensity of scale energy extracted/released along their route, $\partial \phi_{r_{x}} / \partial r_{x}+\partial \phi_{r_{y}} / \partial r_{y}+\partial \phi_{z_{c}} / \partial z_{c}$. The iso-surface in panel $(a)$ reports the energy-containing region of the augmented space of scales and positions, $\left\langle\delta q^{2}\right\rangle=0.98\left\langle\delta q^{2}\right\rangle_{\max }$. In panels $(b)$ and $(c)$ two lateral views are reported together with the iso-contours of the source term $\xi$ evaluated in the planes $r_{y}=0$ and $r_{x}=0$, respectively.

spatial fluxes. In figure 8 the behaviours of the total spatial flux $\phi_{z_{c}}$, the turbulent spatial flux $\phi_{z_{c}}^{\text {turb }}$ and the pressure spatial flux $\phi_{z_{c}}^{\text {press }}$ are shown in the $\left(r_{y}, r_{z}\right)=(0,0)$ plane. In accordance with the single-point budgets shown in $\S 4$, the pressure flux is negative, $\phi_{z_{c}}^{\text {press }}<0$, for the entire jet width, $z_{c}^{*}<170$, and positive, $\phi_{z_{c}}^{\text {press }}>0$, only in the external transitional region of the jet, $z_{c}^{*}>170$; see the dashed line in figure 8(c). The maximum of the inward pressure flux is reached at the energy-containing scales, $r^{*}=O(130)$. On the other hand, the turbulent spatial flux spreads from the production region, thus showing an inward flux $\phi_{z_{c}}^{\text {turb }}<0$ for $z_{c}^{*}<40$ and an outward flux $\phi_{z_{c}}^{\text {turb }}>0$ for $z_{c}^{*}>40$; see 

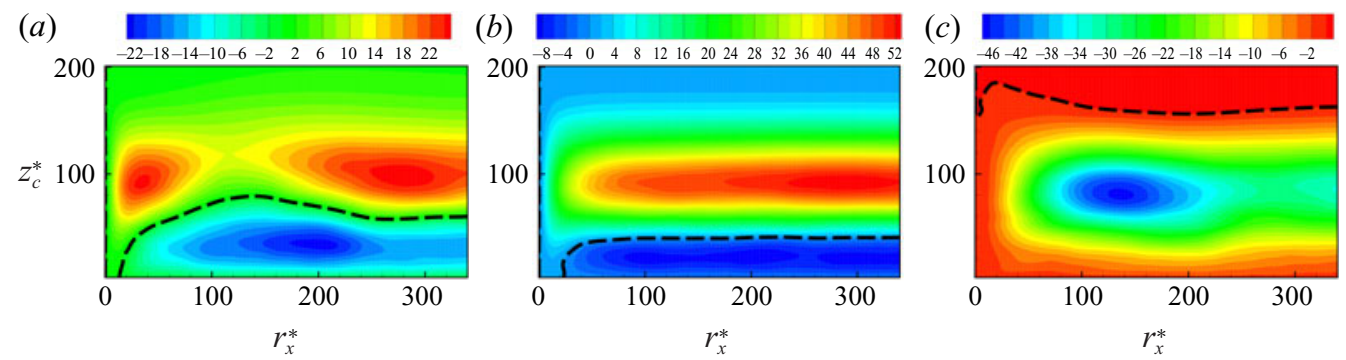

Figure 8. Iso-contours of (a) the total spatial flux $\phi_{z_{c}},(b)$ the spatial turbulent flux $\phi_{z_{c}}^{\text {turb }}$ and $(c)$ the spatial pressure flux $\phi_{z_{c}}^{\text {press }}$ in the plane $\left(r_{y}, r_{z}\right)=(0,0)$. In all the panels, the dashed line reports the null iso-level of the corresponding spatial flux, i.e. $\phi_{z_{c}}=0$ in $(a), \phi_{z_{c}}^{\text {turb }}=0$ in $(b)$ and $\phi_{z_{c}}^{\text {press }}=0$ in $(c)$.

figure $8(b)$. The spatial turbulent flux is active for a wide range of scales encompassing both inertial and energy-containing scales, contrary to the pressure flux where a peak activity is measured for the energy-containing scales $r^{*}=O(130)$. Hence, (i) the turbulent flux is more active than the pressure flux at inertial and dissipative scales. Furthermore, (ii) the turbulent flux is found to be positive at small dissipative scales, $r^{*}<O(20)$, for the entire jet width, also in the inner region where, for larger scales, is directed towards the jet centreline; see the dashed line in figure $8(b)$. The combined effect of these two aspect leads to a total spatial flux $\phi_{z_{c}} \approx \phi_{z_{c}}^{\text {turb }}+\phi_{z_{c}}^{\text {press }}$ being $\phi_{z_{c}}^{\text {visc }} \approx 0$ almost everywhere, with three peak activities; see figure $8(a)$. Two peaks of outward flux centred at $z_{c}^{*}=100$ involving separately small $r^{*}=O(30)$ and very large scales $r^{*}=O(280)$. One peak activity of inward flux centred at $z_{c}^{*}=30$ sustaining the large scales $r^{*}=O(200)$ of the inner region of the jet. On the other hand, the small dissipative scales of the entire jet width $r^{*}<O(20)$ are involved in an outward flux, thus explaining the observed ascending direct energy cascade feeding the $z_{c}$-distributed dissipative range of small scales in figure 7 .

\subsubsection{Family of fluxes $\mathcal{B}$}

The second family of fluxes is that feeding the inner region of the jet. This field of fluxes diverges from the singularity point moving towards smaller spanwise scales, $\phi_{r_{y}}<0$, and larger streamwise scales, $\phi_{r_{x}}>0$, while slightly ascending towards the outer regions, $\phi_{z_{c}}>0$. After this initial part of the paths, the field of fluxes bends towards inner locations, $\phi_{z_{c}}<0$, and, while maintaining a flow towards smaller spanwise scales $\phi_{r_{y}}<0$, show a spread towards both larger and smaller streamwise scales, $\phi_{r_{x}}>0$ and $\phi_{r_{x}}<0$, respectively. As better shown in figure 7(b), this spreading occurs for streamwise scales of the order of $r_{x}^{*}=O(150)$. This scenario is maintained down to the jet centreline where fluxes get progressively aligned with the plane of scales $\left(r_{x}, r_{y}\right)$ since, for $z_{c}=0$, the spatial flux is null for obvious statistical symmetry reasons, $\phi_{z_{c}}=0$, and only cascade mechanisms in the space of scales $\left(\phi_{r_{x}}, \phi_{r_{x}}\right)$ survive. As shown by the intensity of the flux divergence reported with colours in figure 7, the field of fluxes extracts scale energy from the large energy-containing scales of the turbulence production region I and releases it to narrower scales in the jet inner region II characterized by both larger and smaller streamwise lengths. Hence, the field of fluxes while descending towards the inner region conforms with the Richardson picture of turbulence cascade towards small $\left(r_{x}, r_{y}\right)$-scales for $r_{x}^{*}<O(150)$, thus feeding the dissipative range of small scales of the jet centreline in conjunction with the branch of fluxes $\mathcal{A}$. On the other hand, for $r_{x}^{*}>O(150)$, the field of fluxes is characterized by reverse cascade mechanisms towards longer scales. 


\section{A. Cimarelli and others}

\subsubsection{Family of fluxes $\mathcal{C}$}

We consider now the family of fluxes flowing from the large energy-containing scales of the production region I towards the outer regions of the jet; see figure 7 . The field of fluxes diverge from the source region towards both longer and shorter streamwise scales, $\phi_{r_{x}}>0$ and $\phi_{r_{x}}<0$, respectively, while intercepting smaller spanwise scales $\phi_{r_{y}}<0$. In accordance with the behaviour of the spatial flux shown in figure 8 , the branch of fluxes diverging towards shorter streamwise scales $\phi_{r_{x}}<0$ is first directed towards the inner region $\phi_{z_{c}}<0$ before eventually bending towards outer positions $\phi_{z_{c}}>0$; see figure $7(b)$ for a better view. On the contrary, the branch of fluxes spreading towards longer streamwise scales $\phi_{r_{x}}>0$ always maintains an outward direction $\phi_{z_{c}}>0$. After this initial spreading, both branches of fluxes while moving towards the outer regions perform reverse cascade phenomena towards longer and wider fluctuations, $\phi_{r_{x}}>0$ and $\phi_{r_{y}}>0$, respectively; see both figures $7(b)$ and $7(c)$. This scenario is maintained up the jet border, since, for $z_{c}^{*}>180$, the fluxes become asymptotically aligned with the vertical direction, i.e. the cascade mechanisms in the space of scales are no more relevant in the external region IV of the jet, $\left(\phi_{r_{x}}, \phi_{r_{y}}\right) \approx(0,0)$.

As shown by the intensity of the flux divergence reported with colours in figure 7 , the family of fluxes $\mathcal{C}$ along their path extracts scale energy in the production region $\mathrm{I}$ and through reverse cascade mechanisms releases it to sustain longer and wider fluctuations of the outer region III. The question now is, how is scale energy dissipated in the outer and external regions of the jets? Indeed, after the reverse cascade mechanisms of the outer region, the fluxes align to the vertical direction in the external region. Hence, no bending towards the dissipative range of small scales is observed. Clearly, the mechanisms of dissipation of the scale energy carried by this field of fluxes are not those associated with the Kolmogorov picture of turbulence. An answer to this issue is given in $\S 5.3$ based on some features of the hyper-plane $\left(r_{x}=0, t=120\right)$ which is addressed in the following section.

\subsection{The hyper-plane $\left(r_{x}=0, t=120\right)$}

The trajectories of the flux vector field, $\left(\phi_{r_{y}}, \phi_{r_{z}}, \phi_{z_{c}}\right)$, coloured by the intensity of scale energy extracted/released along their route, $\partial \phi_{r_{y}} / \partial r_{y}+\partial \phi_{r_{z}} / \partial r_{z}+\partial \phi_{z_{c}} / \partial z_{c}$ are shown in figure 9. The plots show that the origin of the field of fluxes is a line of the augmented space of scales and positions spanning different spanwise scales $r_{y}$ and located at $z_{c}^{*}=60$ for $r_{z}=0$. As shown by the iso-surface of $\left\langle\delta q^{2}\right\rangle=0.98\left\langle\delta q^{2}\right\rangle_{\max }$ in figure $9(a)$ and by the iso-contours of the source term $\xi$ in figure $9(b)$, this source line is located within the energy-containing and turbulence production region of the augmented space. Hence, the field of fluxes takes origin from the source region of the hyper-plane $r_{x}=0$ and diverge exhibiting a well-defined scale-energy path which can be divided into three different groups whose distinguishing feature is that of the sink regions eventually sustained.

\subsubsection{Family of fluxes $\mathcal{A}$}

The first family of fluxes is that feeding the $z_{c}$-distributed dissipative range of small scales. This family of fluxes departs from the source line for spanwise scales $r_{y}^{*}<100$ and divides into two branches: one moving towards the outer regions $\phi_{z_{c}}>0$ and the other towards the inner part of the jet $\phi_{z_{c}}<0$. For both branches, the departure from the source line is given by a reverse scale-energy flux towards larger cross-flow scales while maintaining a forward flux towards smaller spanwise scales, $\phi_{r_{z}}>0$ and $\phi_{r_{y}}<0$, respectively; see figure $9(c)$ for a better view. The fluxes then bend towards smaller cross-flow scales, thus forming a direct 
(a)

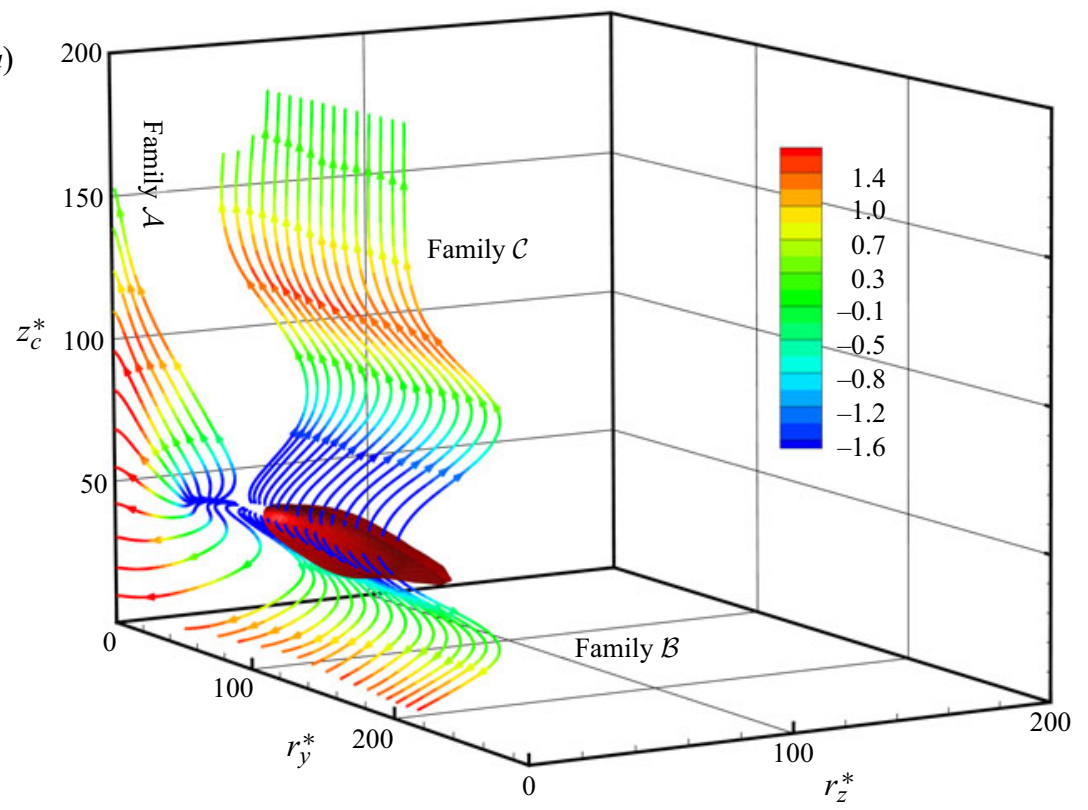

(b)

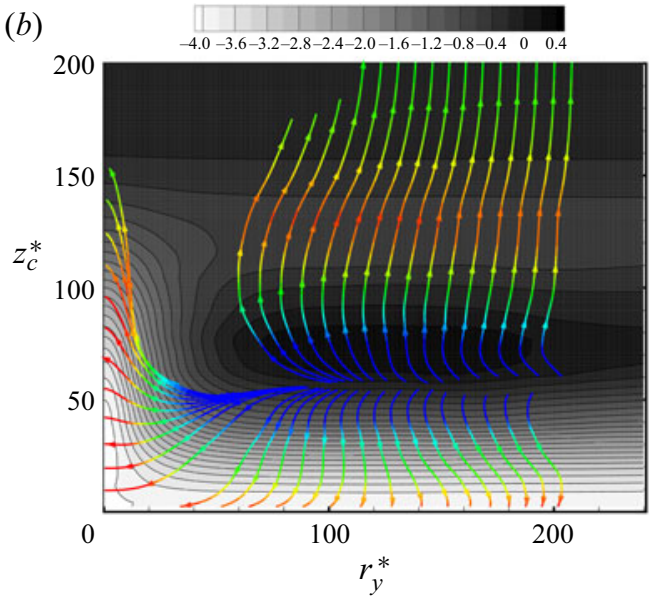

(c)

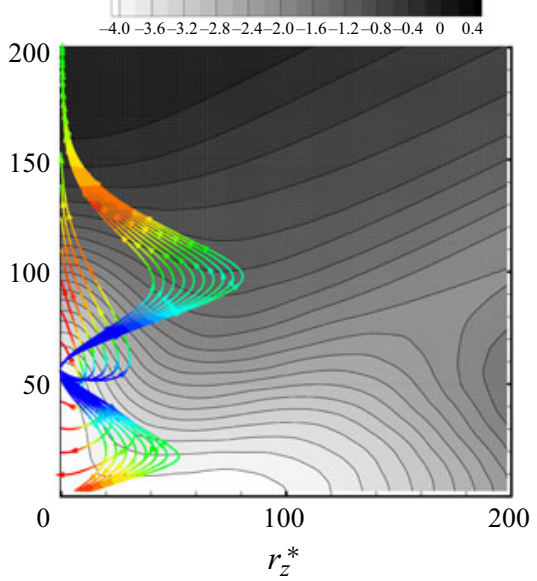

Figure 9. Paths of scale energy in the hyper-plane $r_{x}=0$. Trajectories of the flux vector field, $\left(\phi_{r_{y}}, \phi_{r_{z}}, \phi_{z_{c}}\right)$, coloured by the intensity of scale energy extracted/released along their route, $\partial \phi_{r_{y}} / \partial r_{y}+\partial \phi_{r_{z}} / \partial r_{z}+\partial \phi_{z_{c}} / \partial z_{c}$. The iso-surface in panel $(a)$ reports the energy-containing region of the augmented space of scales and positions, $\left\langle\delta q^{2}\right\rangle=0.98\left\langle\delta q^{2}\right\rangle_{\max }$. In panels $(b, c)$ two lateral views are reported together with the iso-contours of the source term $\xi$ evaluated in the planes $r_{z}=0$ and $r_{y}=0$, respectively.

forward cascade, $\phi_{r_{z}}<0$ and $\phi_{r_{y}}<0$, while maintaining an ascending/descending spatial flux, respectively, for the two branches. The paths are finally closed in the dissipative range of small scales where the spatial flux becomes negligible, thus satisfying the small-scale asymptotic, $\left(\phi_{r_{y}}, \phi_{r_{z}}, \phi_{z_{c}}\right) \sim(1,1,0) r^{*}$ (Cimarelli et al. 2013). As shown with colours in figure 9 , the fluxes are found to extract scale energy in the first part of the path and to release it in the final part. The scenario is that of scale energy extracted from a variety of spanwise and cross-flow scales in the range $r_{y}^{*} \in(40,100)$ and $r_{z}^{*} \in(0,40)$ of the production region I and released to the $z_{c}$-distributed dissipative range of small scales by 


\section{A. Cimarelli and others}

means of forward cascade processes. Hence, the present view is entirely consistent with the picture of the turbulence cascade.

\subsubsection{Family of fluxes $\mathcal{B}$}

The second family of fluxes is that feeding the inner region of the jet. The fluxes depart from the source line for very large spanwise scales $r_{y}^{*}>100$ by performing a reverse flux towards taller fluctuations, $\phi_{r_{z}}>0$, while descending towards the inner regions of the jet $\phi_{z_{c}}<0$; see figure $9(c)$ for a better view. By reaching the jet centreline distance $z_{c}^{*} \approx 30$, the fluxes eventually bend towards smaller cross-flow scales, $\phi_{r_{z}}<0$, and, while maintaining a negative spatial flux $\phi_{z_{c}}<0$, reach the jet centreline region. In this last part of the path, fluxes get progressively aligned with the plane of scales $\left(r_{y}, r_{z}\right)$ since, for $z_{c}=0$, the spatial flux is null for obvious statistical symmetry reasons, $\phi_{z_{c}}=0$, and only cascade mechanisms in the space of scales $\left(\phi_{r_{y}}, \phi_{r_{z}}\right)$ survive. Note that along the entire path, the flux in the spanwise scales $\phi_{r_{y}}$ is almost negligible with respect to that occurring in the cross-flow scales and in physical space, $\phi_{r_{z}}$ and $\phi_{z_{c}}$, respectively. The only exception is the very inner part of the jet for $z_{c}^{*}<10$ where cascade mechanisms also become active in the spanwise scales, thus contributing to the forward cascade sustaining the dissipative range of small scales of the jet centreline. As shown with colours in figure 9, the fluxes are found to extract scale energy in the first part of the path and to release it in the final part. The scenario is that of scale energy extracted from relatively tall and wide fluctuations, $r_{z}^{*} \in(0,40)$ and $r_{y}^{*}>100$, in the production region I and released to small-scale fluctuations in the inner region II by means of forward cascade processes up to the dissipative range.

\subsubsection{Family of fluxes $\mathcal{C}$}

Finally, let us consider the third family of fluxes whose distinguishing feature is that it feeds the outer region of the jet. The fluxes take origin from the source line for very large spanwise scales $r_{y}^{*}>100$. In the first part of the path, the flux performs a reverse transfer towards larger cross-flow scales, $\phi_{r_{z}}>0$, while ascending towards the outer regions of the jet $\phi_{z_{c}}>0$; see figure 9(c) for a better view. After reaching the jet centreline distance $z_{c}^{*} \approx 90$, the fluxes, while ascending, bend towards smaller cross-flow scales $\phi_{r_{z}}<0$. This scenario of forward cascade in the cross-over scales is maintained up to the external region IV of the jet where only spatial fluxes survive since the cascade processes in the space of scales become negligible, $\left(\phi_{r_{y}}, \phi_{r_{z}}\right) \approx(0,0)$, and, hence, the fluxes become asymptotically aligned to the vertical direction. The cascade mechanisms in the spanwise scales are in general weak with respect to those in the cross-flow scales. But a forward cascade towards smaller spanwise scales, $\phi_{r_{y}}<0$, can be recognized in the first part of the path where a reverse cascade in the cross-over scales occurs. On the other hand, a reverse cascade towards wider fluctuations, $\phi_{r_{y}}>0$, is observed in the outer region III of the jet where the flux in the cross-flow scales is, on the contrary, in the forward direction, $\phi_{r_{z}}<0$. As shown with colours in figure 9, the overall picture is that fluxes extract scale energy from fluctuations of the production region I whose widths and heights are $r_{y}^{*}>100$ and $r_{z}^{*} \in(0,40)$ and release it mostly in the outer region III to wider fluctuations whose height is in the range $r_{z}^{*} \in(10,30)$.

\subsection{On the sink regions of the augmented space of turbulence}

Three families of fluxes have been recognized in the previous analysis of the hyper-planes $r_{z}=0$ and $r_{x}=0$. The distinguishing feature is given by the sink regions eventually 


\section{Spatially evolving cascades in temporal planar jets}

sustained being the $z_{c}$-distributed dissipative range of small scales for family $\mathcal{A}$, the inner region of the jet for family $\mathcal{B}$ and the outer regions of the jet for family $\mathcal{C}$. Between these three groups, only the sink region of family $\mathrm{I}$ is in equilibrium with the fluxes sustaining it since $\partial\left\langle\delta q^{2}\right\rangle / \partial t$ asymptotically vanishes in the small-scale limit being $\delta q^{2}=0$ at any time for $\boldsymbol{r}=0$. In other words, the scale energy released by fluxes in the $z_{c}$-distributed dissipative range of small scales is balanced by the local source term $\xi$ that in this range of small scales simply reduces to the turbulent dissipation, $\xi=-4 \tilde{\epsilon}$. On the contrary, this equilibrium condition is not satisfied for the sink regions of the family of fluxes $\mathcal{B}$ and $\mathcal{C}$. In particular, the scale energy released by family $\mathcal{B}$ in the inertial and energy-containing scales of the inner region is not enough to balance the local source term which is negative $\xi<0$, thus leading to a temporal decay of the scale-energy content $\partial\left\langle\delta q^{2}\right\rangle / \partial t<0$. On the contrary, the scale energy released by family $\mathcal{C}$ in the outer regions of the jet exceeds the local source term which is again negative $\xi<0$, thus leading to a temporal growth of the scale-energy content $\partial\left\langle\delta q^{2}\right\rangle / \partial t>0$. This last sink region involves the outer and external loci of the jet (regions III and IV), thus including the interface layer, and deserves further considerations.

The previous analysis of the field of fluxes $\mathcal{C}$ in the hyper-planes $r_{z}=0$ and $r_{x}=0$ revealed that long and wide fluctuations are sustained in the outer region III through spatially ascending reverse cascade mechanisms taking origin from the production region I. Hence, large-scale inertial phenomena are found at the basis of the temporal growth of scale energy $\partial\left\langle\delta q^{2}\right\rangle / \partial t>0$, thus enabling the first stage of cross-flow spreading of the turbulent jet. By entering the external region IV, the cascade mechanisms in the space of horizontal scales asymptotically vanish, $\phi_{r_{x}}=\phi_{r_{y}}=0$, and only a forward cascade in the cross-flow scales survives $\phi_{r_{z}}<0$ while maintaining a positive spatial flux. Hence, in the external region of the jet the overall picture is that of a cross-flow shrinking of the scales intercepted by the ascending large-horizontal-scale fluxes. This very anisotropic shrinkage of the scales constrains turbulence to two dimensions, thus suppressing the cascade picture towards small horizontal scales that is indeed absent in this region of the flow, $\phi_{r_{x}}=\phi_{r_{y}}=0$. Turbulence is then dissipated $\xi<0$ by shearing friction phenomena occurring in a very thin layer of these large-horizontal-scale structures whose thickness of the order of a few Kolmogorov scales decreases during the outward propagation. Since the scale-energy contribution of the fluxes sustaining this thin layer of large-scale motions exceed the local source term, a temporal growth of the scale-energy content $\partial\left\langle\delta q^{2}\right\rangle / \partial t>0$ is performed, thus enabling the second stage of cross-flow spreading of the turbulent jet.

\section{Scale-by-scale budgets}

The unexpected paths taken by the scale-energy flux in the combined physical/scale space, which are a substantial novelty with respect to known descriptions of turbulent jets, may have major repercussions on our theoretical understanding with practical consequences for modelling. Let us try to grasp the essential aspects by means of a more quantitative scale-by-scale analysis. To this aim, we fix the streamwise and cross-flow scales, $r_{x}=$ $r_{z}=0$, and we consider separately the scale-by-scale features of the four relevant flow regions identified so far by also fixing the distance from the jet centreline. This type of study, while enabling a more quantitative view of the flow, lacks for the description of its multi-dimensionality and of its scale-space anisotropy that, however, have been addressed in the previous $\S 5$ and, when relevant, will be explicitly retrieved. The symbolic form of the generalized Kolmogorov equation reported in (3.12) will be considered, here reported 


\section{A. Cimarelli and others}

for clarity,

$$
\frac{\partial\left\langle\delta q^{2}\right\rangle}{\partial t}=T_{r}+D_{r}+T_{z}+\xi
$$

where $T_{r}=T_{r_{x}}+T_{r_{y}}+T_{r_{z}}=-\partial\left(\phi_{r_{i}}^{\text {turb }}+\phi_{r_{i}}^{\text {mean }}\right) / \partial r_{i}$ is the inertial scale-space transport term, $D_{r}=D_{r_{x}}+D_{r_{y}}+D_{r_{z}}=-\partial \phi_{r_{i}}^{v i s c} / \partial r_{i}$ is the viscous scale-space transport term, $T_{z}=T_{z}^{\text {turb }}+T_{z}^{\text {visc }}+T_{z}^{\text {press }}$ is the spatial transport term with its turbulent, viscous and pressure contributions and, finally, $\xi=\Pi-4 \tilde{\epsilon}$ is the source term, balance of production and dissipation.

\subsection{Viscous and inertial subrange scaling in the turbulent jet}

Before starting the scale-by-scale analysis, it is worth addressing the inertial and viscous scaling when non-equilibrium effects are present such as that given by the non-steady state of the temporal jet. By considering statistical isotropy, the generalized Kolmogorov equation written in spherical coordinates reads as

$$
\frac{\partial\left\langle\delta q^{2}\right\rangle}{\partial t}+\frac{1}{r^{2}} \frac{\partial}{\partial r}\left(r^{2} \phi_{r_{\|}}\right)=-4 \tilde{\epsilon},
$$

where $r$ is the scale-space radial coordinate and

$$
\phi_{r_{\|}}=\left\langle\delta q^{2} \delta u_{\|}\right\rangle-2 v \frac{\partial\left\langle\delta q^{2}\right\rangle}{\partial r}=\phi_{r_{\|}}^{\text {turb }}+\phi_{r_{\|}}^{v i s c}
$$

is the radial scale-energy flux with $\delta u_{\|}$the longitudinal velocity increment. By integrating (6.2), we finally obtain an equation for the scale-energy flux:

$$
\phi_{r_{||}}=-\frac{4}{3} \tilde{\epsilon} r-\frac{1}{r^{2}} \int r^{2} \frac{\partial\left\langle\delta q^{2}\right\rangle}{\partial t} \mathrm{~d} r .
$$

The first term in (6.4) is the classical inertial and viscous subrange scaling while the second term is the non-equilibrium correction. Indeed, in the case of statistical steady conditions, (6.4) leads to the following relations:

$$
\begin{gathered}
\phi_{r_{\mid}} \approx-2 \nu \frac{\partial\left\langle\delta q^{2}\right\rangle}{\partial r}=-\frac{4}{3} \tilde{\epsilon} r \quad\left\langle\delta q^{2}\right\rangle=\frac{1}{3} \frac{\tilde{\epsilon}}{v} r^{2} \quad \text { for } r=O(\eta), \\
\phi_{r_{||}} \approx\left\langle\delta q^{2} \delta u_{\| \mid}\right\rangle=-\frac{4}{3} \tilde{\epsilon} r \quad\left\langle\delta q^{2}\right\rangle=\left(\frac{4}{3} \tilde{\epsilon}\right)^{2 / 3} r^{2 / 3} \quad \text { for } r>O(\eta) .
\end{gathered}
$$

Let us quantify the role of non-equilibrium in (6.4) in order to predict the induced deviation from the classical scaling. By assuming a complete flow self-preservation, we can write the second-order moment of the velocity increment as

$$
\left\langle\delta q^{2}\right\rangle=\left\langle\delta q^{2}\right\rangle(r, t)=\mathcal{U}^{2}(t) f(\hat{r}), \quad \hat{r}=\frac{r}{\mathcal{H}(t)},
$$

where $\mathcal{U}(t)$ and $\mathcal{H}(t)$ are jet characteristic velocity and length scales. This self-similarity assumption allows us to decompose the time derivative term as

$$
\frac{\partial\left\langle\delta q^{2}\right\rangle}{\partial t}=2 \mathcal{U} f \frac{\mathrm{d} \mathcal{U}}{\mathrm{d} t}+\mathcal{U}^{2} \frac{\partial f}{\partial \hat{r}} \frac{\partial \hat{r}}{\partial t} .
$$

Self-similarity also allows us to write the standard entrainment parametrisation $\mathrm{d} \mathcal{H} / \mathrm{d} t=$ $\alpha \mathcal{U}$, where $\alpha$ is the entrainment coefficient. Furthermore, note that the volume flux is 


\section{Spatially evolving cascades in temporal planar jets}

conserved in the temporal jet implying that $\mathcal{H} \mathrm{d} \mathcal{U} / \mathrm{d} t=-\mathcal{U} \mathrm{d} \mathcal{H} / \mathrm{d} t$. Hence, after some manipulations, the self-similar decomposition of the temporal decay becomes

$$
\frac{\partial\left\langle\delta q^{2}\right\rangle}{\partial t}=-\alpha \frac{\mathcal{U}^{3}}{\mathcal{H}}\left(f+\frac{\partial f \hat{r}}{\partial \hat{r}}\right)
$$

and the non-equilbrium correction term of (6.4) can be rewritten as

$$
\frac{1}{r^{2}} \int_{r} r^{2} \frac{\partial\left\langle\delta q^{2}\right\rangle}{\partial t} \mathrm{~d} r=-\frac{\mathcal{U}^{3}}{\mathcal{H}} \frac{\alpha}{r^{2}} \int_{r} r^{2}\left(f+\frac{\partial f \hat{r}}{\partial \hat{r}}\right) \mathrm{d} r .
$$

Assuming now a scale-space scaling in the form of a power law,

$$
f(\hat{r})=A \hat{r}^{\beta},
$$

we can finally quantify the non-equilibrium term as

$$
\frac{1}{r^{2}} \int_{r} r^{2} \frac{\partial\left\langle\delta q^{2}\right\rangle}{\partial t} \mathrm{~d} r=-A \mathcal{U}^{3} \frac{\alpha(2+\beta)}{3+\beta}\left(\frac{r}{\mathcal{H}}\right)^{1+\beta} .
$$

By inserting (6.12) into (6.4) and by using again self-similarity also for dissipation, $\tilde{\epsilon}=B \mathcal{U}^{3} / \mathcal{H}$, we can finally estimate the balance for the scale-energy flux as

$$
\phi_{r_{\|}}=\mathcal{U}^{3}\left[-\frac{4}{3} B\left(\frac{r}{\mathcal{H}}\right)+A \frac{\alpha(2+\beta)}{3+\beta}\left(\frac{r}{\mathcal{H}}\right)^{1+\beta}\right],
$$

thus highlighting that, for any $\beta>0$, the effect on the scaling of the scale-energy flux of the second term due to non-equilibrium can be neglected only for very small scales, $r=O(\eta)$.

\subsection{Production region I}

We start the scale-by-scale analysis by considering the production region I. In figure $10(a)$ the generalized Kolmogorov equation evaluated in the $\left(0, r_{y}, 0,60\right)$-space using a semi-log plot is shown. The range of large scales is sustained by local turbulence production mechanisms $\Pi$. Scale energy available at large scales is then drained by the inertial scale-space transport $T_{r}<0$ to initiate cascade mechanisms towards small scales and by the spatial transport $T_{z}<0$ to feed the outer and inner regions of the jet. The scale energy extracted by $T_{r}$ and $T_{z}$ together with the local dissipation mechanisms is larger than that produced, thus leading to a temporal decay of the scale-energy content of the large scales, $\partial\left\langle\delta q^{2}\right\rangle / \partial t<0$. At intermediate scales, the turbulence production and the spatial transport decrease their intensity while the inertial scale-space transport becomes positive, $T_{r}>0$, thus leaving space to an inertial subrange of scales sustained by turbulent cascade mechanisms. Also in this case, the scale energy provided by the cascade is not enough to balance that extracted by the spatial transport and by the local dissipation, thus leading to a temporal decay, $\partial\left\langle\delta q^{2}\right\rangle / \partial t<0$. This scenario is closed at small scales where anisotropic and inhomogeneous processes are negligible, $\Pi \approx T_{z} \approx 0$, and the viscous scale-space transport $D_{r}>0$ becomes dominant, thus releasing the scale energy provided by the inertial scale-space transport $T_{r}$ to the smallest scales of the flow. In this case the scale energy provided by $D_{r}$ balances with dissipation,thus reaching a small-scale equilibrium, $\partial\left\langle\delta q^{2}\right\rangle / \partial t=0$. Overall, a classical picture of turbulence where scale energy is introduced 


\section{A. Cimarelli and others}
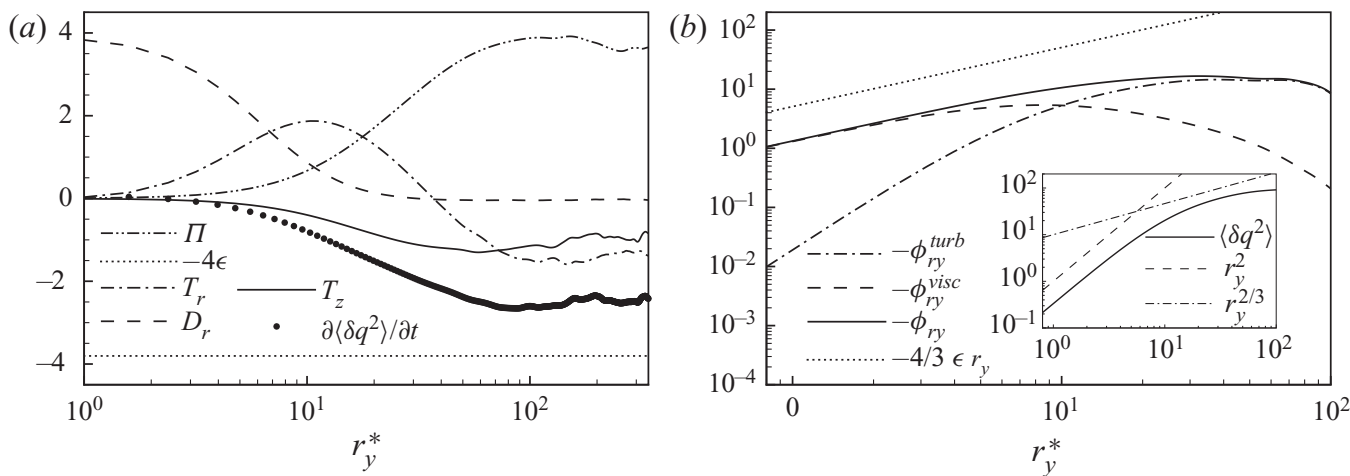

Figure 10. Scale-by-scale processes in the production region I, $z^{*}=60$. (a) Generalized Kolmogorov equation evaluated in the $\left(0, r_{y}, 0,60\right)$-space using a semi-log plot. Turbulence production $\Pi$ (dashed-dotted-dotted line), turbulent dissipation $-4 \tilde{\epsilon}$ (dotted line), inertial scale transport $T_{r}$ (dashed-dotted line), viscous scale transport $D_{r}$ (dashed line), spatial transport $T_{z}$ (solid line) and time variation of scale energy $\partial\left\langle\delta q^{2}\right\rangle / \partial t$ (circle). (b) Scale-space fluxes in the $\left(0, r_{y}, 0,60\right)$-space using a log-log plot: $-\phi_{r_{y}}^{\text {turb }}$ (dashed-dotted line), $-\phi_{r_{y}}^{\text {visc }}$

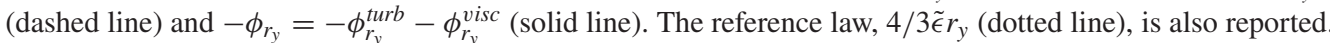
Inset panel: scale energy $\left\langle\delta q^{2}\right\rangle$ (solid line), $r^{2}$ (dashed line) and $r^{2 / 3}$ (dashed-dotted line).

at large scales and then transferred to small scales where it is eventually dissipated by viscosity is observed in the production region.

A more detailed analysis of the budget reveals the following reduced descriptions of the flow:

$$
\begin{gathered}
\partial\left\langle\delta q^{2}\right\rangle / \partial t \approx \Pi+T_{r_{z}}+T_{z}^{\text {turb }}+T_{z}^{\text {press }}-4 \tilde{\epsilon}<0 \text { for } r^{*}>60 \\
\partial\left\langle\delta q^{2}\right\rangle / \partial t \approx \Pi+T_{r}+T_{z}^{\text {turb }}-4 \tilde{\epsilon}<0 \text { for } 6<r^{*}<60 \\
0 \approx D_{r}-4 \tilde{\epsilon} \text { for } r^{*}<6
\end{gathered}
$$

thus highlighting that, at large scales $r^{*}>60$, (6.14), the scale energy draining is predominantly performed by the turbulent spatial transport, $T_{z}^{\text {turb }}<0$, and by the cross-flow component of the divergence of the scale-space transport, $T_{r_{z}}<0$. On the contrary, the pressure spatial transport together with turbulence production are responsible for sustaining the large-scale motion, $T_{z}^{\text {press }}>0$ and $\Pi>0$. At intermediate scales, (6.15), only the spatial turbulent transport $T_{z}^{\text {turb }}$ survives since $T_{z}^{\text {press }} \approx 0$ and all the components of the scale-space transport become active, $T_{r}=T_{r_{x}}+T_{r_{y}}+T_{r_{z}}$. Finally, at the smallest scales of the flow $r^{*}<6,(6.16)$, a balance between scale-space viscous transport and dissipation is observed, thus leading to a small-scale equilibrium range, $\partial\left\langle\delta q^{2}\right\rangle / \partial t=0$, in accordance with the reasoning reported in $\S 6.1$. As shown in figure $10(b)$, this aspect leads to a recover of the classical viscous scaling of turbulence, $\phi_{r_{y}}^{v i s c} \sim r_{y}$ and $\left\langle\delta q^{2}\right\rangle \sim r_{y}^{2}$ for $r^{*}<6$ while, at intermediate scales $6<r^{*}<60$, the inertial scaling of turbulence, $\phi_{r_{y}}^{\text {turb }} \sim r_{y}$ and $\left\langle\delta q^{2}\right\rangle \sim r_{y}^{2 / 3}$, is affected by deviations induced by the non-negligible effects of anisotropic production, inhomogeneous spatial fluxes and non-equilibrium; see the reduced budget (6.15). Overall, the total scale-space flux roughly follows the linear law, $\phi_{r_{y}}=\phi_{r_{y}}^{\text {turb }}+\phi_{r_{y}}^{\text {visc }} \sim r_{y}$ for $r^{*}<20$. 
(a)

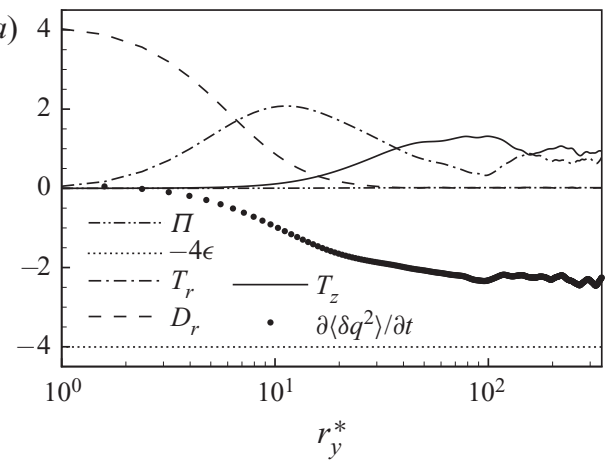

(b)

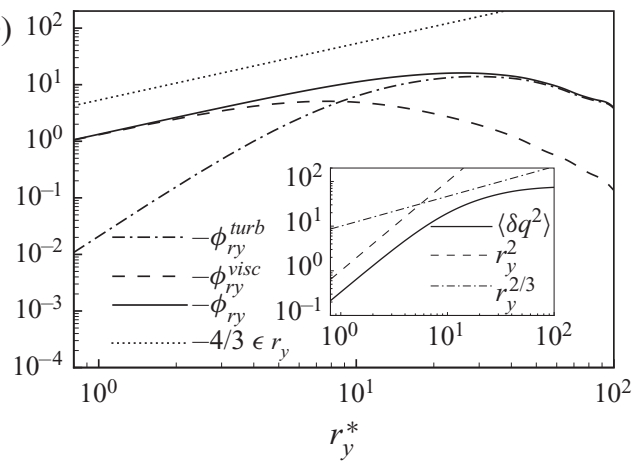

Figure 11. Scale-by-scale processes in the inner region II, $z^{*}=0$. See the caption to figure 10.

\subsection{Inner region II}

The scale-by-scale analysis of the inner region is reported in figure 11(a) where the generalized Kolmogorov equation evaluated in the $\left(0, r_{y}, 0,0\right)$-space using a semi-log plot is shown. The overall scenario is similar to that reported for the production region, i.e. scale energy is provided at large scales and then transferred to small scales where it is eventually dissipated by viscosity. The main difference comes from the mechanisms sustaining the large-scale motion. Indeed, contrary to the production region, in the inner region turbulence production is negligible $\Pi \approx 0$ and the large scales are sustained by the spatial transport that turns out to be positive with both its turbulent and pressure components, $T_{z}^{\text {turb }}>0$ and $T_{z}^{\text {press }}>0$.

A more detailed analysis of the budget allows us to write the following reduced descriptions:

$$
\begin{aligned}
\partial\left\langle\delta q^{2}\right\rangle / \partial t \approx T_{r_{z}} & +T_{z}^{\text {turb }}+T_{z}^{\text {press }}-4 \tilde{\epsilon}<0 \text { for } r^{*}>40, \\
\partial\left\langle\delta q^{2}\right\rangle / \partial t & \approx T_{r}-4 \tilde{\epsilon}<0 \text { for } 4<r^{*}<40, \\
0 & \approx D_{r}-4 \tilde{\epsilon} \text { for } r^{*}<4 .
\end{aligned}
$$

Hence, the intermediate range of scales, (6.18), also slightly differs from that of the production region being that both the turbulence production and the spatial transport are negligible. As a consequence, the intermediate range of scales closely resemble the classical scaling of turbulence being affected only by non-equilibrium effects. Accordingly, in figure $11(b)$ the inertial scaling of turbulence, $\phi_{r_{y}}^{t u r b} \sim r_{y}$ and $\left\langle\delta q^{2}\right\rangle \sim$ $r_{y}^{2 / 3}$, is found to be better captured being affected by deviations solely induced by non-equilibrium. On the other hand, as expected from the arguments reported in $\S 6.1$, a balance between dissipation and viscous scale-space transport, (6.19), is observed in the range of small scales. Hence, a recovery of the classical viscous scaling of turbulence, $\phi_{r_{y}}^{\text {visc }} \sim r_{y}$ and $\left\langle\delta q^{2}\right\rangle \sim r_{y}^{2}$ for $r^{*}<4$ is measured; see figure 11(b). Overall, we observe that the total scale-space flux roughly follows the linear law, $\phi_{r_{y}}=\phi_{r_{y}}^{\text {turb }}+\phi_{r_{y}}^{\text {visc }} \sim r_{y}$, for a larger range of scales with respect to the production region for $r^{*}<30$.

\subsection{Outer region III}

In figure $12(a)$ the generalized Kolmogorov equation evaluated in the $\left(0, r_{y}, 0,140\right)$-space is shown. The scale-by-scale budget in the outer region shows a significantly different 


\section{A. Cimarelli and others}
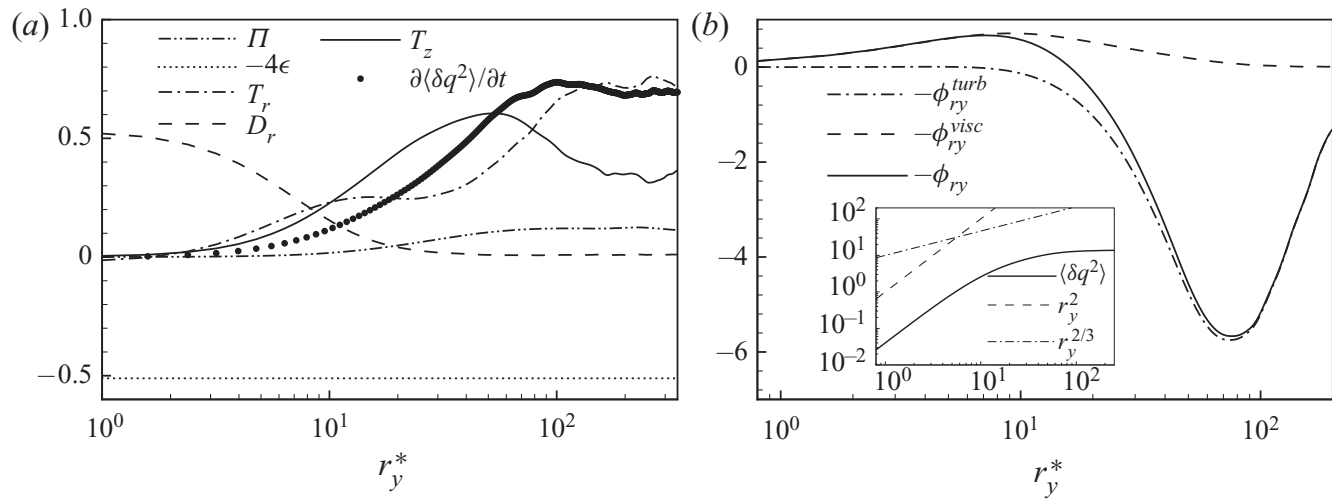

Figure 12. Scale-by-scale processes in the outer region III, $z^{*}=140$. See the caption to figure 10 . The only difference with respect to figure 10 is the use of a semi-log plot in panel $(b)$ due to the presence of reverse cascade phenomena in this region of the flow.

scenario with respect to the production and inner regions. Scale energy is provided by the spatial transport $T_{z}>0$ with a peak of activity at intermediate scales. At larger scales, scale energy provided by the spatial transport is overcome by the activity of the scale-space transport $T_{r}>0$. Overall, the scale energy provided at intermediate and large scales by the spatial and scale-space transports exceeds the local dissipation, thus leading to a temporal growth of the scale-energy content, $\partial\left\langle\delta q^{2}\right\rangle / \partial t>0$. On the other hand, an equilibrium is recovered in the small-scale range being that the scale energy provided by the viscous scale-space transport is in balance with the local turbulent dissipation.

As shown in $\S 5$, this scale energy provided by the scale-space transport term is released at the large scales by reverse cascade phenomena. This mechanism is confirmed by the scale-space flux $\phi_{r_{y}}$ reported in figure $12(b)$ that shows an inversion of sign for $r^{*} \approx 20$, thus performing a reverse transfer towards larger scales for $r^{*}>20$ and a very weak forward transfer towards smaller scales for $r^{*}<20$. In the same panel, both the turbulent and viscous contributions to the scale-space flux are shown, thus highlighting that the reverse transfer is performed by the turbulent flux $-\phi_{r_{y}}^{\text {turb }}<0$ being that the viscous flux is smaller and always directed towards small scales $-\phi_{r_{y}}^{\text {visc }}>0$.

A more detailed study of the budget allows us to write the following reduced descriptions of the outer region:

$$
\begin{gathered}
\partial\left\langle\delta q^{2}\right\rangle / \partial t \approx T_{r_{z}}+T_{z}^{\text {turb }}+T_{z}^{\text {press }}-4 \tilde{\epsilon}>0 \text { for } r^{*}>100, \\
\partial\left\langle\delta q^{2}\right\rangle / \partial t \approx T_{r}+T_{z}^{\text {turb }}-4 \tilde{\epsilon}>0 \text { for } 10<r^{*}<100, \\
0 \approx D_{r}-4 \tilde{\epsilon} \text { for } r^{*}<10 .
\end{gathered}
$$

The first important aspect to highlight concerns the large-scale behaviour for $r^{*}>100$, (6.20). Indeed, the scale energy provided by the spatial flux $T_{z} \approx T_{z}^{t u r b}+T_{z}^{\text {press }}$ is entirely due to the turbulent component $T_{z}^{t u r b}>0$ being that the pressure contribution is negative $T_{z}^{\text {press }}<0$. In accordance with the analysis of the turbulent kinetic budget and of the scale-energy paths, $\S \S 4$ and 5 , the pressure flux extracts energy in the outer region to feed the production and inner regions from one side and the external region from the other. As unequivocally shown here in quantitative terms, the pressure transport activity is concentrated at scales larger than those of the turbulent transport; see (6.20) and (6.21). The second important aspect concerns the intermediate scales for $10<r^{*}<100,(6.21)$. 
(a)

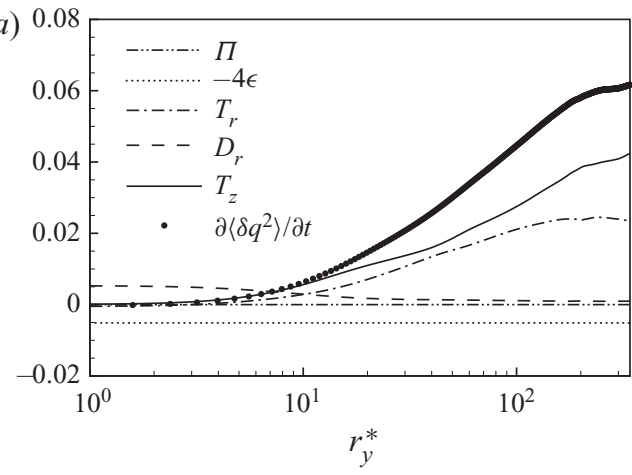

(b)

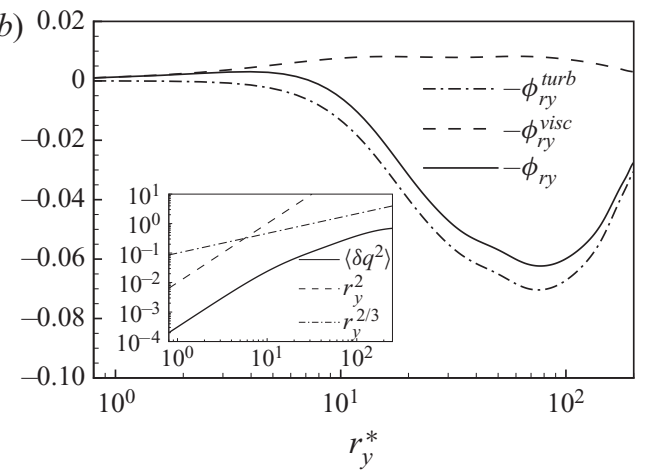

Figure 13. Scale-by-scale processes in the interface region, $z^{*}=200$. See the caption to figure 10 . The only difference with respect to figure 10 is the use of a semi-log plot in panel $(b)$ due to the presence of reverse cascade phenomena in this region of the flow.

In this range of scales, the scale energy provided by the scale-space transport, $T_{r}=$ $T_{r_{x}}+T_{r_{y}}+T_{r_{z}}>0$ is entirely due to the cross-flow component, $T_{r_{z}}>0$, being that the other two components are negative, $T_{r_{x}}<0$ and $T_{r_{y}}<0$. Indeed, in accordance with the scale-energy paths analysis reported in $\S 5$, within this range of scales, the horizontal components of the scale-space transport extract scale energy to feed the reverse cascade towards larger horizontal scales while a forward transfer towards smaller cross-flow scales is observed at every scale.

The overall picture of the jet growth in the outer region is that turbulent fluctuations at intermediate and large scales are sustained by complex phenomena such as inhomogeneous spatial fluxes and reverse cascade mechanisms. This phenomenology is in contrast with the classical notion of turbulence and may have repercussions on both theoretical and modelling approaches. In accordance with (6.22), classical phenomena are recovered only at very small scales where, as shown in figure 12(b), the viscous scaling, $\phi_{r_{y}}^{v i s c} \sim r_{y}$ and $\left\langle\delta q^{2}\right\rangle \sim r_{y}^{2}$ for $r^{*}<10$ are measured.

\subsection{External region IV}

We now consider the scale-by-scale features of the external flow region. We recall that the external region has been defined as the region of the flow involving distances from the centreline $z_{c}^{*}>180$, and, hence, it is located outside the jet interface evaluated at $z_{c}^{*}=h_{\Omega}^{*}=171$ by using the rule of $2 \%$ of the jet centreline enstrophy. The generalized Kolmogorov equation evaluated in the $\left(0, r_{y}, 0,200\right)$-space is shown in figure 13(a). Scale energy is found to be provided at intermediate and large scales by the spatial transport $T_{z}>0$ and by the turbulent scale-space transport $T_{r}>0$. This feeding largely exceeds the local dissipation phenomena, thus leading to a temporal growth of the scale-energy content in this region, $\partial\left\langle\delta q^{2}\right\rangle / \partial t>0$. On the contrary, a balance between viscous scale-space transport and dissipation occurs at small scales.

A more detailed study of the budget reveals interesting phenomena as highlighted by the following reduced descriptions:

$$
\begin{gathered}
\partial\left\langle\delta q^{2}\right\rangle / \partial t \approx T_{r_{z}}+T_{z}^{\text {turb }}+T_{z}^{\text {press }}>0 \quad \text { for } r^{*}>100 \\
\partial\left\langle\delta q^{2}\right\rangle / \partial t \approx T_{r_{z}}+T_{z}^{\text {turb }}>0 \quad \text { for } 6<r^{*}<100, \\
0 \approx D_{r}-4 \tilde{\epsilon} \quad \text { for } r^{*}<6 .
\end{gathered}
$$




\section{A. Cimarelli and others}

As shown by the reduced budgets (6.23) and (6.24), for almost the entire range of scales with exception to the smallest ones $\left(r^{*}<6\right)$, turbulent dissipation $\tilde{\epsilon}$ is not anymore a fundamental quantity. This aspect highlights that this region of the flow is actually a non-turbulent region. Accordingly, the intensity of the velocity fluctuations is weak and cascade mechanisms in the space of horizontal scales are absent. Indeed, only a forward transfer in the space of cross-over scales survives, $T_{r} \approx T_{r_{z}}$; see again the reduced budgets (6.23) and (6.24). Hence, scale energy is sustained at very large horizontal scales and small cross-flow scales. In accordance with the scale-energy paths reported in $\S 5$, the final stage of propagation of the turbulent jet, $\partial\left\langle\delta q^{2}\right\rangle / \partial t>0$, occurs in a very thin layer of large-horizontal-scale structures moving outward from the jet itself. As shown by the reduced budget (6.23), at very large scales this outward transfer is sustained by both pressure and turbulent spatial transports, $T_{z} \approx T_{z}^{\text {turb }}+T_{z}^{\text {press }}>0$, while at intermediate scales the strength of the pressure term decreases and only the contribution of the turbulent transport survives, $T_{z} \approx T_{z}^{\text {turb }}>0$; see the reduced budget (6.24).

In accordance with the turbulent kinetic energy budget analysed in $\S 4$, the combined role played by the turbulent and pressure spatial transport changes by increasing the distance from the jet centreline. Even if not shown for the sake of brevity, the reduced budgets $(6.23),(6.24)$ and $(6.25)$ are found to asymptotically reduce to the following behaviour:

$$
\partial\left\langle\delta q^{2}\right\rangle / \partial t=T_{z}^{\text {press }} \forall r^{*}
$$

by increasing the jet centreline distance $z_{c}$. By recalling that the pressure transport is active at very large scales and that the external region is populated by non-turbulent fluctuations, we argue that the correlation of pressure and vertical velocity fluctuations $\left\langle\delta p^{\prime} \delta w^{\prime}\right\rangle=2\left\langle p^{\prime} w^{\prime}\right\rangle-\left\langle p^{\prime}\left(\boldsymbol{x}^{\prime \prime}\right) w^{\prime}\left(\boldsymbol{x}^{\prime}\right)\right\rangle-\left\langle w^{\prime}\left(\boldsymbol{x}^{\prime \prime}\right) p^{\prime}\left(\boldsymbol{x}^{\prime}\right)\right\rangle$ at the basis of the pressure transport term is a result of a non-turbulent large-scale phenomenon. In particular, we conjecture that the vertical displacement of almost quiescent fluid in the external region is given by pressure-mediated non-local interactions induced by the streamwise advection inside the jet of a large-scale spanwise vortex pattern reminiscent of the Kelvin-Helmholtz instability. This large-scale and non-local pressure energy transfer feed fluctuations in the external region that are non-turbulent in nature but represent the triggering mechanisms of transitional phenomena that in time would lead to turbulent fluctuations and, hence, to the growth of the jet width.

\section{Conclusions}

The processes of entrainment and mixing in free-shear flows are known to be characterized by a variety of scales ranging from the large-scale fluctuations of the turbulent core to the small-scale motions acting on its convoluted surface boundary. The understanding of the interaction mechanisms between this variety of scales and positions is a challenge for theories and turbulence closures. To address this issue, the theoretical framework of the generalized Kolmogorov equation is here developed and applied to the statistical symmetries of a turbulent planar temporal jet. The formalism is based on the second-order moment of the two-point velocity increment. The structure of the equation, in the form of a divergence of a scale-energy flux, allows us for the first time to reconcile the large- and small-scale features of the turbulent flow and of the turbulent entrainment by tracing the scale-energy paths in the augmented space of scales and positions. The emerging picture is the following.

The field of fluxes arises from the large energy-containing scales of the production region $\left(z^{*} \approx 60\right)$. Scale energy in this region of the augmented space of scales and 


\section{Spatially evolving cascades in temporal planar jets}

positions is also made available by local turbulence production mechanisms that exceed the local turbulent dissipation, $\xi>0$. The field of fluxes while radiating from this source region are also energized along their path. The amount of scale energy extracted exceeds the local source mechanisms, thus leading to a scale-energy decay, $\partial\left\langle\delta q^{2}\right\rangle / \partial t<0$. The fluxes emerging from the source region diverge exhibiting a well-defined scale-energy path which can be divided into three groups whose distinguishing feature is that of the attractor, the sink of the augmented space, where the fluxes release scale energy and are eventually absorbed.

(i) The first family of fluxes $\mathcal{A}$, while emanating from the source region, bends towards smaller scales performing an energy cascade sustaining the $z_{c}$-distributed dissipative range of small scales. Scale energy released by fluxes in this sink region is balanced by the local dissipation processes, thus recovering a statistical equilibrium, $\partial\left\langle\delta q^{2}\right\rangle / \partial t=0$. The concurrent role of pressure and turbulent spatial fluxes is such that this family of fluxes along their paths firstly descends towards the inner region and subsequently bend at small scales attaining an ascending behaviour.

(ii) The second family of fluxes $\mathcal{B}$ releases scale energy extracted in the source region to sustain the turbulent fluctuations of the inner region. The scale energy released by fluxes in this region of the flow does not balance the local negative source mechanisms, $\xi<0$, thus leading to a scale-energy decay, $\partial\left\langle\delta q^{2}\right\rangle / \partial t<0$. A detailed analysis of this family reveals the presence of a cross-over scale of the order of the jet half-width $r^{*}=O(150)=O\left(h_{\Omega}\right)$. For smaller scales, the fluxes perform a forward cascade, thus sustaining smaller scales in the inner region. On the other hand, for larger scales, a reverse cascade is observed in the streamwise scales, thus sustaining longer fluctuations in the inner region.

The overall picture of both the production and inner regions of the jet conforms with the classical description of turbulence as far as we consider scales smaller than the jet half-width $r^{*}<O(150)=O\left(h_{\Omega}\right)$. Scale energy is introduced at large scales by source mechanisms in the production region and spatial fluxes in the inner region and transferred to smaller scales before being dissipated by viscous mechanisms. From a theoretical point of view, this scenario satisfies the reduced descriptions

$$
\begin{aligned}
\partial\left\langle\delta q^{2}\right\rangle / \partial t & \approx T_{r}-4 \tilde{\epsilon}<0 \text { for } 4<r^{*}<40, \\
0 & \approx D_{r}-4 \tilde{\epsilon} \text { for } r^{*}<4,
\end{aligned}
$$

thus highlighting that the classical viscous scaling, $\phi_{r_{\|}} \sim r$ and $\left\langle\delta q^{2}\right\rangle \sim r^{2}$ for $r=O(\eta)$, is recovered while the classical inertial scaling, $\phi_{r_{\|}} \sim r$ and $\left\langle\delta q^{2}\right\rangle \sim r^{2 / 3}$ for $r>O(\eta)$, is actually influenced by non-equilibrium effects.

(iii) The third family of fluxes $\mathcal{C}$ characterizes the outer and external regions of the jet. The scale energy extracted from the source region is released mostly in the outer region of the jet through spatially ascending reverse cascade processes. The picture is that of long and wide fluctuations generated by inertial reverse cascade mechanisms originating from the smaller energy-containing scales of the production region. Scale energy provided by fluxes exceeds the local negative source mechanisms, $\xi<0$, and leads to a growth of the scale-energy content, $\partial\left\langle\delta q^{2}\right\rangle / \partial t>0$, thus establishing the first stage of cross-flow spreading of the jet. Note that this scenario is entirely consistent with the spectral enstrophy balance analysis conducted in Cimarelli et al. (2015a). By entering the external region of the jet, the reverse cascade mechanisms in the space of horizontal scales asymptotically vanish and only a spatially ascending forward transfer towards smaller cross-flow scales survives. The overall scenario is that of ascending large-horizontal-scale motions transferring their energy to smaller and smaller cross-flow 


\section{A. Cimarelli and others}

scales. Scale energy also does not cascade down in the horizontal scales, thus supporting the idea that turbulence is constrained to two dimensions and that viscous dissipation is given by shearing friction phenomena involving a thin layer of large-horizontal-scale motions. Scale energy provided by fluxes in this thin layer exceeds that extracted by friction phenomena, thus enabling the last stage of propagation of the turbulent jet, $\partial\left\langle\delta q^{2}\right\rangle / \partial t>0$.

The complex nature of the flux and source phenomena described in the outer and external regions of the jet are challenges for theories and turbulence closures. In the outer region where most of the scale energy is released to sustain the temporal growth, reverse cascade mechanisms should be taken into account to represent the enlargement of flow structures. From a theoretical point of view, the reverse cascade scenario satisfies the reduced descriptions

$$
\begin{gathered}
\partial\left\langle\delta q^{2}\right\rangle / \partial t \approx T_{r_{z}}+T_{z}^{t u r b}+T_{z}^{\text {press }}-4 \tilde{\epsilon}>0 \text { for } r^{*}>100, \\
\partial\left\langle\delta q^{2}\right\rangle / \partial t \approx T_{r}+T_{z}^{t u r b}-4 \tilde{\epsilon}>0 \text { for } 10<r^{*}<100
\end{gathered}
$$

and no inertial scaling is observed. On the other hand, in the external region turbulent dissipation cannot be used anymore as a fundamental quantity and very anisotropic transfer processes should be considered. A reduced description of this region is

$$
\partial\left\langle\delta q^{2}\right\rangle / \partial t \approx T_{r_{z}}+T_{z}^{\text {turb }}+T_{z}^{\text {press }}>0 \text { for } r^{*}>100,
$$

that by increasing the jet centreline distance asymptotically reduces to

$$
\partial\left\langle\delta q^{2}\right\rangle / \partial t=T_{z}^{\text {press }}>0 \quad \forall r^{*} .
$$

We argue that this final asymptotic behaviour is given by a non-turbulent large-scale phenomenon of vertical displacement of almost quiescent fluid due to the streamwise advection of the large-scale spanwise vortex pattern of the jet reminiscent of the Kelvin-Helmholtz instability. This pressure-mediated phenomenon represents the triggering mechanism of transitional phenomena that in time would contribute to the growth of turbulent fluctuations in the very external region of the jet.

Acknowledgements. The authors acknowledge the financial support of the Engineering and Physical Sciences Research Council (EPSRC) through the project 'Multi-scale dynamics at the turbulent/non-turbulent interface of jets and plumes' - grant EP/R042640/1. Computing time has been provided by the UK National Supercomputing Service ARCHER and by the Supercomputing Wales facility. The authors also acknowledge Dr A. Fregni for assistance in the revision of the article. We finally would like to acknowledge Professor C. M. Casciola for the interesting discussions we had on our work.

Declaration of interests. The authors report no conflict of interest.
Author ORCIDs.
(ㄱ) A. Cimarelli http://orcid.org/0000-0001-5165-9639;
(1) J.-P. Mollicone http://orcid.org/0000-0002-8989-4762;
(D) M. van Reeuwijk http://orcid.org/0000-0003-4840-5050;
(ㄱ) E. De Angelis http://orcid.org/0000-0002-9183-2413.

\section{Appendix A. Scaling in the self-similar regime}

By following van Reeuwijk \& Holzner (2014), the self-similar regime of a temporal planar jet is characterized by a temporal behaviour of the characteristic velocities which is proportional to $t^{-1 / 2}$ and of the characteristic lengths which is proportional to $t^{1 / 2}$, where 
(a)

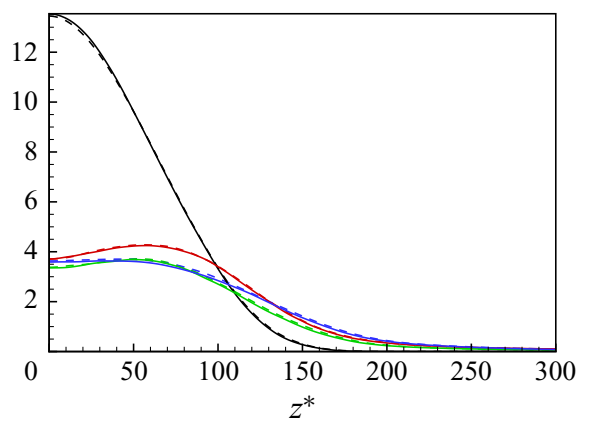

(b)

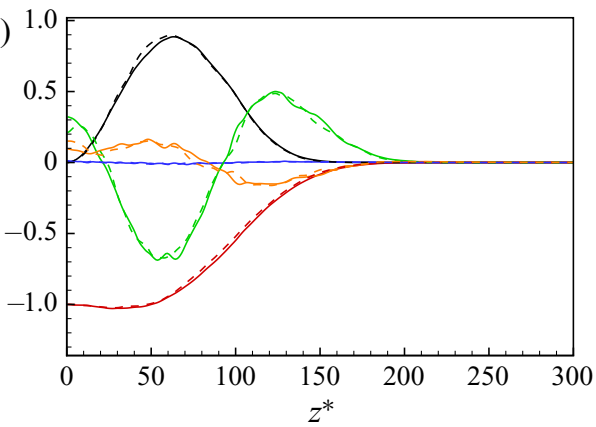

Figure 14. (a) Mean and fluctuating velocity profiles scaled in Kolmogorov units and reported for two different times within the self-similar regime, $t=120$ (solid line) and $t=160$ (dashed line); $U^{*}\left(z^{*}\right)$ (black), $\sqrt{\left\langle u^{\prime} u^{\prime}\right\rangle^{*}}\left(z^{*}\right)$ (red), $\sqrt{\left\langle v^{\prime} v^{\prime}\right\rangle^{*}}\left(z^{*}\right)$ (green) and $\sqrt{\left\langle w^{\prime} w^{\prime}\right\rangle^{*}}\left(z^{*}\right)$ (blue). (b) Turbulent kinetic energy budget scaled in Kolmogorov units and reported for two different times within the self-similar regime, $t=120$ (dashed line) and $t=160$ (solid line); turbulence production (black), dissipation (red), turbulent transport (green), pressure transport (orange) and viscous diffusion (blue).

$t$ is time. As shown in figure 2, such scaling is confirmed by the present direct numerical simulation data. Particularly relevant is the scaling of the centreline turbulent dissipation, $\epsilon_{c l} \sim 1 / t^{2}$, which can be inferred by considering dissipation as the cube of a velocity scale over a length scale. It is possible now to build a self-similar velocity and length scale that solely depend on the self-similar scaling of dissipation. These characteristic scales are the Kolmogorov scales

$$
\begin{gathered}
\eta(\operatorname{Re}, t)=\left(\frac{\nu^{3}}{\epsilon_{c l}}\right)^{1 / 4}=A(\operatorname{Re}) t^{1 / 2}, \\
u_{\eta}(\operatorname{Re}, t)=\left(\nu \epsilon_{c l}\right)^{1 / 4}=B(\operatorname{Re}) t^{-1 / 2},
\end{gathered}
$$

where $A$ and $B$ are Reynolds dependent constants and the time dependence of $\eta$ and $u_{\eta}$ is entirely absorbed by dissipation. To verify the accuracy of the scaling of flow variables with the Kolmogorov scales, in figure 14 the mean and fluctuating velocity profiles (a) and the turbulent kinetic energy budget $(b)$ scaled in Kolmogorov units denoted with the superscript $*$ is shown for two different stages of evolution of the flow within the self-similar regime $t=120$ and $t=160$. The agreement is remarkable thus showing the degree of self-preservation of the flow considered. The quality of the scaling is so high that, in principle, can also be used to improve the statistical convergence of the data by also averaging between different times once the data are made dimensionless with the Kolmogorov scales.

It is finally worth mentioning that the Reynolds number remains constant during the decay of the temporal jet and, as a consequence, the ratio between macro- and micro-scales also remains constant. This peculiar aspect of the flow considered here suggests that the time behaviour of the Kolmogorov scales proportionally follows that of the macro-scale such as the volume flux and the characteristic jet half-width.

\section{REFERENCES}

Burattini, P., Antonia, R.A. \& Danaila, L. 2005 Scale-by-scale energy budget on the axis of a turbulent round jet. J. Turbul. 6, N19.

Cimarelli, A., AвBÀ, A. \& Germano, M. 2019 General formalism for a reduced description and modelling of momentum and energy transfer in turbulence. J. Fluid Mech. 866, 865-896. 


\section{A. Cimarelli and others}

Cimarelli, A., Cocconi, G., Frohnapfel, B. \& De Angelis, E. $2015 a$ Spectral enstrophy budget in a shear-less flow with turbulent/non-turbulent interface. Phys. Fluids 27, 125106.

Cimarelli, A. \& DE Angelis, E. 2012 Anisotropic dynamics and sub-grid energy transfer in wall-turbulence. Phys. Fluids 24, 015102.

Cimarelli, A. \& DE Angelis, E. 2014 The physics of energy transfer toward improved subgrid-scale models. Phys. Fluids 26, 055103.

Cimarelli, A., De Angelis, E. \& Casciola, C.M. 2013 Paths of energy in turbulent channel flows. J. Fluid Mech. 715, 436-451.

Cimarelli, A., De Angelis, E., Jimenez, J. \& Casciola, C.M. 2016 Cascades and wall-normal fluxes in turbulent channel flows. J. Fluid Mech. 796, 417-436.

Cimarelli, A., De Angelis, E., Schlatter, P., Brethouwer, G., Talamelli, A. \& Casciola, C.M. $2015 b$ Sources and fluxes of scale energy in the overlap layer of wall turbulence. J. Fluid Mech. 771, 407-423.

Corrsin, S. \& Kistler, A.L. 1955 The free-stream boundaries of turbulent flows. NACA Tech. Rep. TN-1244.

Craske, J. \& VAn ReEuwiJk, M. 2015 Energy dispersion in turbulent jets. Part 1. Direct simulation of steady and unsteady jets. J. Fluid Mech. 763, 500-537.

Danaila, L., Anselmet, F., Zhou, T. \& AntoniA, R.A. 2001 Turbulent energy scale budget equations in a fully developed channel flow. J. Fluid Mech. 430, 87-109.

Djenidi, L., Antonia, R.A., Lefeuvre, N. \& Lemay, J. 2016 Complete self-preservation on the axis of a turbulent round jet. J. Fluid Mech. 790, 57-70.

Dubrulle, B. 2019 Beyond Kolmogorov cascades. J. Fluid Mech. 867, P1.

Germano, M. 2007a A direct relation between the filtered subgrid stress and the second order structure function. Phys. Fluids 19, 038102.

Germano, M. $2007 b$ The elementary energy transfer between the two-point velocity mean and difference. Phys. Fluids 19,085105.

Gomes-Fernandes, R., GAnAPATHisubramani, B. \& VASsilicos, J.C. 2015 The energy cascade in near-field non-homogeneous non-isotropic turbulence. J. Fluid Mech. 771, 676-705.

HiLl, R.J. 2002 Exact second-order structure-function relationship. J. Fluid Mech. 468, 317-326.

Hussain, A.K.M.F. 1986 Coherent structures and turbulence. J. Fluid Mech. 173, 303-356.

Kolmogorov, A.N. 1991a Dissipation of energy in locally isotropic turbulence. Proc. R. Soc. Lond. A 434, 15-17 (reprinted from Dokl. Akad. Nauk SSSR, 1941, vol. 32(1)).

Kolmogorov, A.N. $1991 b$ The local structure of turbulence in incompressible viscous fluid for very large Reynolds number. Proc. R. Soc. Lond. A 434, 9-13 (reprinted from Dokl. Akad. Nauk SSSR, 1941, vol. 30(4)).

Marati, N., Casciola, C.M. \& Piva, R. 2004 Energy cascade and spatial fluxes in wall turbulence. J. Fluid Mech. 521, 191-215.

Mollicone, J.P., Battista, F., Gualtieri, P. \& CAsciola, C.M. 2018 Turbulence dynamics in separated flows: the generalised Kolmogorov equation for inhomogeneous anisotropic conditions. J. Fluid Mech. 841, 1012-1039.

Portela, F.A., PAPAdAkis, G. \& VAssilicos, J.C. 2017 The turbulence cascade in the near wake of a square prism. J. Fluid Mech. 825, 315-352.

Redford, J.A., CAStro, I.P. \& Coleman, G.N. 2012 On the universality of turbulent axisymmetric wakes. J. Fluid Mech. 710, 419-452.

VAN ReEuwijk, M. \& Holzner, M. 2014 The turbulence boundary of a temporal jet. J. Fluid Mech. 739, 254-275.

Rincon, F. 2006 Anisotropy, inhomogeneity and inertial-range scalings in turbulent convection. J. Fluid Mech. 563, 43-69.

Sadeghi, H., LAvoie, P. \& Pollard, A. 2016 Scale-by-scale budget equation and its self-preservation in the shear-layer of a free round jet. Intl J. Heat Fluid Flow 61, 85-95.

Saikrishnan, N., De Angelis, E., Longmire, E.K., Marusic, I., Casciola, C.M. \& Piva, R. 2012 Reynolds number effects on scale energy balance in wall turbulence. Phys. Fluids 24 (1), 015101.

DA Silva, C.B., Hunt, J.C.R., EAmes, I. \& Westerweel, J. 2014 Interfacial layers between regions of different turbulence intensity. Annu. Rev. Fluid Mech. 46, 567-590.

DA Silva, C.B. \& MÈTAIS, O. 2002 On the influence of coherent structures upon interscale interactions in turbulent plane jets. J. Fluid Mech. 473, 103-145.

DA Silva, C.B. \& PEREIRA, J.C.F. 2008 Invariants of the velocity-gradient, rate-of-strain, and rate-of-rotation tensors across the turbulent/nonturbulent interface in jets. Phys. Fluids 20, 055101. 


\section{Spatially evolving cascades in temporal planar jets}

Sreenivasan, K.R., Ramshankar, R. \& Meneveau, C. 1989 Mixing, entrainment and fractal dimensions of surfaces in turbulent flows. Proc. R. Soc. Lond. A 421, 79-108.

Togni, R., Cimarelli, A. \& De Angelis, E. 2015 Physical and scale-by-scale analysis of Rayleigh-Bénard convection. J. Fluid Mech. 782, 380-404.

Togni, R., Cimarelli, A. \& De Angelis, E. 2019 Resolved and subgrid dynamics of Rayleigh-Bénard convection. J. Fluid Mech. 867, 906-933.

VerstapPen, R.W.C.P. \& Veldman, A.E.P. 2003 Symmetry-preserving discretization of turbulent flows. J. Comput. Phys. 187, 343-368. 